\title{
A Phase field method for modelling anodic dissolution induced stress corrosion crack propagation
}

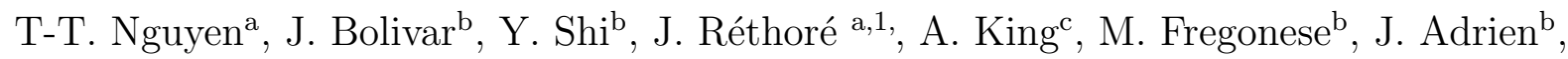 \\ J-Y. Buffiere ${ }^{\mathrm{b}}$, M-C. Baietto ${ }^{\mathrm{a}}$ \\ ${ }^{a}$ Université de Lyon, CNRS, INSA-Lyon, LaMCoS UMR5259, France \\ ${ }^{b}$ Université de Lyon, CNRS, INSA-Lyon, Université Lyon 1, MateIS UMR5510, France \\ ${ }^{c}$ Synchrotron Soleil, Psyche beamline, L'Orme des Merisiers, 91190 Saint Aubin, France
}

\begin{abstract}
The phase field method is a powerful tool for studying microstructural evolution in various domains of material sciences, including phase change, initiation and propagation of fracture. In this work, a new formulation is developed based on the phase field method for modeling stress corrosion cracking (SCC) induced by anodic dissolution. This method was applied for modelling SCC of an aluminum alloy (2xxx series) in a saline medium $(\mathrm{NaCl})$, which allows considering the effects of both electrochemical and mechanical processes. The classical phase transition model for material dissolution is coupled with the mechanical problem in a robust manner, providing an efficient tool for studying the competition between electrochemical and mechanical contributions to fracture. A numerical implementation based on finite elements is elaborated. The numerical results are compared to experimental data obtained by in situ microtomography.

Keywords: Stress corrosion cracking, anodic dissolution, crack propagation, phase field
\end{abstract}

\section{Introduction}

Stress-corrosion cracking (SCC) is a common failure mechanism characterized by a combination of a mechanical load, a corrosive environment and a susceptible material. The occurrence of SCC induces premature failure of the material, which is recognized as potentially dangerous. In that context, a detailed investigation regarding SCC role is necessary to accurately predict the strength and failure of materials and structures.

\footnotetext{
Email address: julien.rethore@ec-nantes.fr (J. Réthoré )

${ }^{1}$ Now at GEM, CNRS UMR 6183 CNRS / Ecole Centrale de Nantes / Université de Nantes
} 
Various mechanisms have been proposed in the literature to explain the synergistic stress-corrosion, such as adsorption model, film rupture model, pre-existing active path model, and embrittlement model,...(see e.g. [1] for a detailed review). Among them, the mechanisms based on the local anodic dissolution of fresh metal created by slip bands emergence at the crack tip [2] is often regarded as one of the most model. It has thus long been recognized as an indispensable step in the production of pure metal salts, energy production in batteries, etc. As a consequence, developing numerical model to study its effect in terms of key engineering parameters is highly required.

A first group of methods based on the moving of sharp interface have been proposed to this aim. In these methods, the interface is represented explicitly to apply the boundary conditions. Finite Element Method (FEM) by using re-meshing technique is widely used within this context [3]. However, the solution is mesh dependent. The remeshing step at each loading step reveals to be computationally expensive in practice and becomes extremely difficult for complex material and/or 3D problems, see e.g. [4] for a detailed review. As a consequence, the Arbitrary Lagrangian-Eulerian (ALE) method [3], has been proposed as an advanced FEM technique. ALE technique shows some improvements in comparison with classical FEM, such as its ability to simulate rapid dissolution processes as in the case of pitting corrosion. This method gives a stable solution when the pit growth is modelled in the work of [5], or [6]. Another alternative technique based on Finite Volume Method (FVM) is proposed in the work of [7] to study localized corrosion. In this work, a voxel method is used to represent the anodic front. The ionic transport and complete electrostatic problems are solved in the FVM context. However, lack of corrosion progresses due to the changes in the electronic nature of boundaries (from anode to cathode and vice versa) is the drawback of this method.

Some other contributions in the context of sharp interface methods are: the work of [8] to study SCC, in which the average current density (computed from the transport equation) is used as a criterion to model the crack growth. However the shape of the crack front must be predefined and it is difficult to simulate complex dissolution morphologies. The level-set technique [9], or cellular automata [10], are also used in order to handle the complex shape of corrosion surfaces. Moreover, in these works, the electrochemical 
problem is often simplified.

Recently, the phase field method, where the sharp interface is described by a smeared interface, has been proposed [11, 12, 13, 14, 15, 16]. This method becomes an efficient tool for the simulation of microstructural evolution in material science. With this method, the explicit tracking of interfaces is not required, i.e. all numerical difficulties encountered in the sharp-interface models are avoided. Therefore, the phase field method is capable to handle arbitrarily complex geometries, even in 3D. The phase field model has been used to investigate the effects of SCC in many works, such as in [17] to model the hydrogeninduced stress-corrosion cracking, or in [18] to account the effects of diffusion of chloride ions in hardened cement paste. The application of this model can also be found in [19] to simulate the corrosion kinetics under a dual-oxidant atmosphere, or to investigate the crack propagation in brittle materials induced by the variations of concentration (electrolyte- $\mathrm{LiFePO}_{4}$ ) in the work [20]

The application of the phase field method to simulate material dissolution can be found in the work of [21]. The authors have proposed a quantitative scheme, where the phase transformation is controlled by multicomponent diffusion. This model has been applied to study the dissolution in Ti-Al-V alloy. In the work of [22], the authors use a phase field model to investigate the dissolution of primary particles in aluminum phase under isothermal diffusion-controlled processes. The dynamics of liquid-solid interfaces resulting from precipitation and/or dissolution processing is studied by [23] by using the phase field method. In the work by [24], SCC induced crack propagation is studied, however, the electrochemical contribution is simplified and not investigated in details. More recently in the work by [25, 26], this method is applied to simulate the pitting corrosion. However, a method to describe all aspects of electrochemical and mechanical processes inducing material dissolution or fracture growth is still missing.

This work started from ideas presented in the work of [25, 27] where the KKS (KimKim-Suzuki) model [11] is adopted with new normalization procedures to describe the dissolution processes (phase transformation from solid to liquid). We propose herein a coupling with mechanical equilibrium to model dissolution induced SCC. It is worth mentioning that, differing from [26], the mechanical process is here directly accounted in 
the free energy functional, providing a performance scheme to investigate the competition between electrochemical and mechanical contribution. We also adopt a robust numerical framework by using the unilateral contact formulation and history functional following [16, 28]. This ensures that the dissolution can be accelerated under tensile mechanical states, and that effect of material microstructure can be considered as mentioned in [24]. It is expected that the proposed model can simulate both the anisotropy in the elastic behaviour and in fracture evolution in the case of SCC.

The outline of the paper is as follows: first some fundamental basics of the model are introduced in Section 2 where several assumptions will be proposed. In Section 3 , a new phase field framework able to model effects of both electrochemical and mechanical processes on crack growth is proposed. Then, the details of the numerical implementation are presented in Sections 4 and 5. Finally, the performances of the new model are illustrated through several numerical examples, which are compared to recent experimental observations in Section 6 .

\section{Fundamentals of the proposed method}

Let $\Omega \subset \mathbb{R}^{D}$ be an open domain describing a corrosion system, which generally contains three domains: (i) metal or solid phase; (ii) electrolyte solution or liquid phase; (iii) an interfacial region where the corrosion occurs.

The characteristic of first metal solid domain is described by temporally and spatially constant metal atom concentration $c_{\text {solid }}^{\text {alloy }}$ That is identified based on its chemical composition. For the example of an alloy, it is composed from chemical element $\mathrm{Me}_{i}$ with molar masses $\mathcal{M}_{i}$, regarding to be identical with molar masses of corresponding ions $\mathrm{Me}_{\mathrm{i}}^{\mathrm{n}+}$. Denoted $f_{\mathcal{M}, i}[\%]$, the mole fraction of species $i$, sometimes also referred to as "molar fraction" is computed by the following:

$$
f_{\mathcal{M}, i}=\frac{f_{m, i} / \mathcal{M}_{i}}{\sum_{j} f_{m, i} / \mathcal{M}_{i}},
$$

where $f_{m, i}[\%]$ is mass fraction of species $i$. Following the work by [29], the average molar mass is computed as:

$$
\overline{\mathcal{M}}=\sum_{i} f_{\mathcal{M}, i} \mathcal{M}_{i}
$$


and the concentration $c_{\text {solid }}^{\text {alloy }}$ of metal atoms in the solid phase now reads:

$$
c_{\text {solid }}^{\text {alloy }}=\frac{\rho_{\text {alloy }}}{\overline{\mathcal{M}}} \text {. }
$$

The corrosion progresses due to the dissolution of Me into aqueous solution as $\mathrm{Me}^{n+}$ ions at the anode boundary. Thus, the anodic reaction involves oxidation of Me as follows:

$$
\mathrm{Me} \rightarrow \mathrm{Me}^{\mathrm{n}+}+\mathrm{ne}^{-}
$$

That provides a diffusional transport of the metal ions from solid domain to liquid domain across the corrosion boundary. As noticed in [30, 29], this corrosion boundary is represented with temporally and spatially constant saturation concentration of dissolved metal ions $c_{s a t}$. This value describes the maximum value of the overall ionic concentration that can be reached $c^{\text {ions }}=\sum_{i} c_{i}^{\text {ions }}$. Actually, due to the diffusional transport of the metal ions, the overall ionic concentration in solution increases until a saturation exists with respect to the chloride or the hydroxychloride complex of the cations in the case of a saline solution, i.e. until chemical equilibrium between the ionic solution and the solid metal is achieved. This leads to the formation of a salt film with saturation concentration $c_{\text {sat }}$. After that, the evolution of corrosion is then controlled by the diffusion of metal ions away from the corrosion boundary (diffusion-controlled corrosion). In case of instantaneous dissolution of a metal alloy, dissolution takes place by orders of magnitude faster than the diffusion of dissolved ions, so that the metal ion concentration remains constantly at the saturation limit $c_{\text {sat }}$, i.e. chemical equilibrium is always achieved at the electrode boundary.

According to the Rankine-Hugoniot condition, the rate of the metal dissolution depends on the ions concentration and its gradient along the interfacial region. This is usually described by the equilibrium between the dissolved metal atoms flux and the velocity of the moving interface [29]. In this work, we use the phase field method to model the dissolution of a metal which is corroding. The corrosion system is now described by a phase field variable $\phi . \phi=1$ is solid phase, $\phi=0$ is liquid phase, and the corrosion boundary (sharp interface) is replaced by a thin interface within the range $0<\phi<1$, (it is often chosen that $\phi=0.05-0.95$ defines transition zone). The corrosion process 
is then simulated by the evolution of auxiliary field $\phi$ over the entire system. The effect of the ions diffusion induced metal dissolution is modeled following the work by [25] who introduce the normalized concentration:

$$
c=\frac{c^{\text {ions }}}{c_{\text {solid }}^{\text {alloy }}}
$$

The ions (or atoms) concentration now reads $c_{s}$ for metal solid and $c_{l}$ for electrolyte, which are defined in the normalized context by the following:

$$
c_{s}=\frac{c_{s}^{\text {alloy }}}{c_{\text {solid }}^{\text {alloy }}} \quad \text { and } \quad c_{l}=\frac{c_{l}^{\text {solution }}}{c_{\text {solid }}^{\text {alloy }}}
$$

where $c_{s}^{\text {alloy }}, c_{l}^{\text {solution }}$ are the real concentration values of solid metal and solution, respectively. The details of this description are depicted in Fig. 1.

The assumption of KKS model [11] is used, in which the transition region is represented as a mixture of the two phases with different chemical compositions. Therefore, the ions concentration at any material point can be described by the following:

$$
c=h(\phi) c_{s}+[1-h(\phi)] c_{l},
$$

where $h(\phi)$ can be understood as the interpolation function, satisfying $h(\phi=0)=0$ to ensure that $c=c_{l}$ in liquid phase, $h(\phi=1)=1$ and $\left.\frac{\partial h(\phi)}{\partial \phi}\right|_{\phi=0, \phi=1}=0$, to ensure that $c=c_{s}$ in solid phase; one choice: $h(\phi)=-2 \phi^{3}+3 \phi^{2}$. This function also plays an important role to describe the loss of material strength discussed in the next section.

In the normalized framework, the metal solid and electrolyte ions concentration, denoted $c_{\text {sat }}^{\text {alloy }}$ and $c_{\text {sat }}^{\text {solution }}$, respectively can read:

$$
c_{s e}=\frac{c_{\text {sat }}^{\text {alloy }}}{c_{\text {solid }}^{\text {alloy }}}=\frac{c_{\text {solid }}^{\text {alloy }}}{c_{\text {solid }}^{\text {alloy }}}=1,
$$

and

$$
c_{l e}=\frac{c_{\text {sat }}^{\text {solution }}}{c_{\text {solid }}^{\text {alloy }}}=\frac{c_{\text {sat }}}{c_{\text {solid }}^{\text {alloy }}},
$$

where $c_{s e}, c_{l e}$ are the saturated concentration of solid and liquid phase after being normalized. The details of the corrosion system in a normalized context is depicted in Fig. 2. 


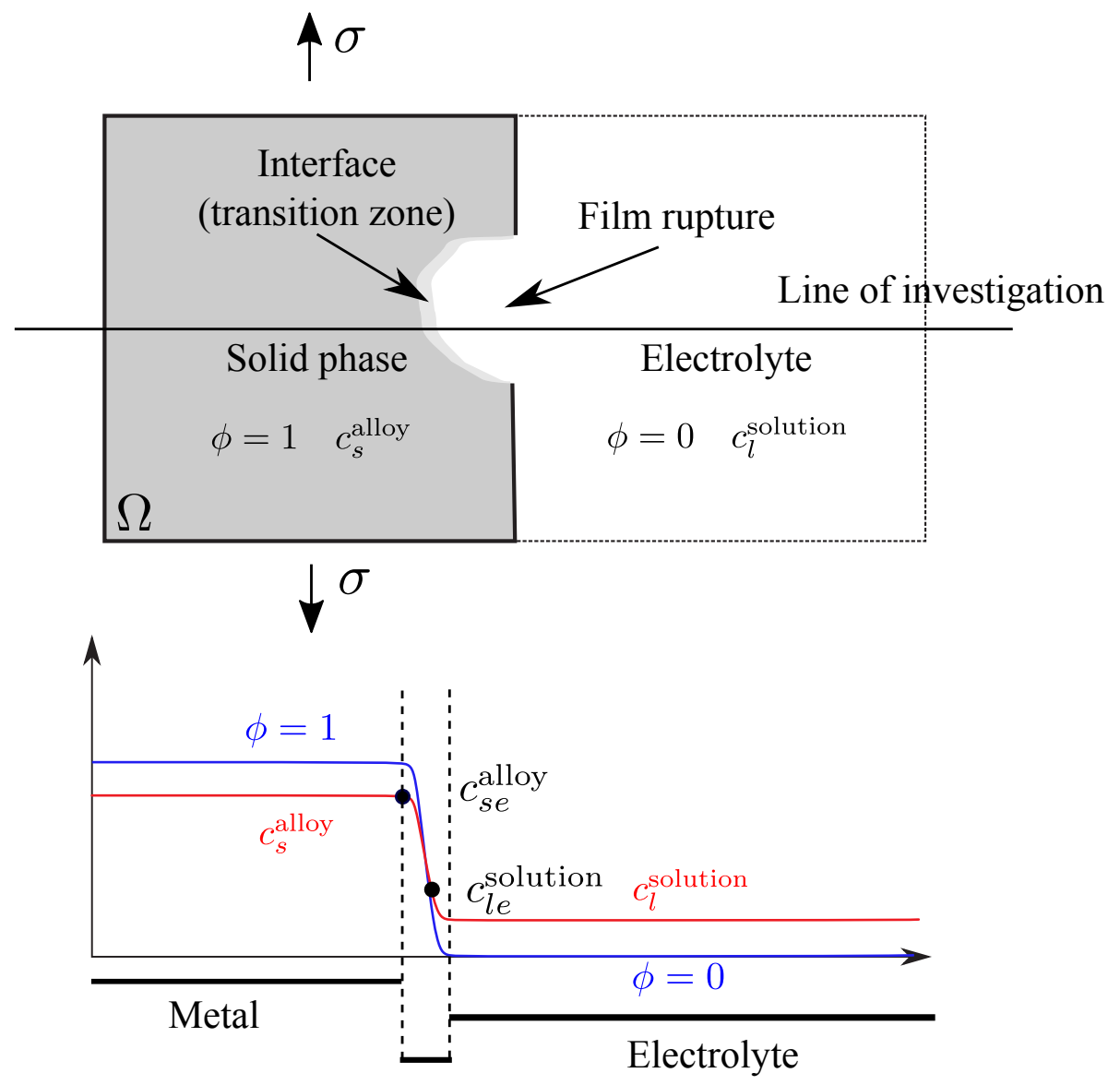

Transition zone

Figure 1: Schematic of a typical corrosion: (top) 2D representation; (bottom) illustration in a line of investigation

In this work, the dissolution of material is not only due to electrochemical processes, but also to mechanical loading. Therefore, the phase field evolution $\phi=1 \rightarrow \phi=0$ represents both interpretations: material dissolution and crack creation. In addition, different schemes based on different diffusion paths are used to describe the breakdown of the passive film, as depicted in Fig. 2, following the work in [31]. In most of situations, the volume of the electrolyte is much larger than the one occupied by the solid metal and where the initiation of SCC occurs. Therefore the ions concentration and phase field in the electrolyte region are assumed to be zero, i.e the film-rupture process is simplified in the numerical model by imposing the Dirichlet boundary conditions: $c=0, \phi=0$ over this region. Otherwise, in the regions where the metal is still covered by passive film, the zero-flux $\frac{\partial c}{\partial n}=0$, and $\frac{\partial \phi}{\partial n}=0$ are imposed. 


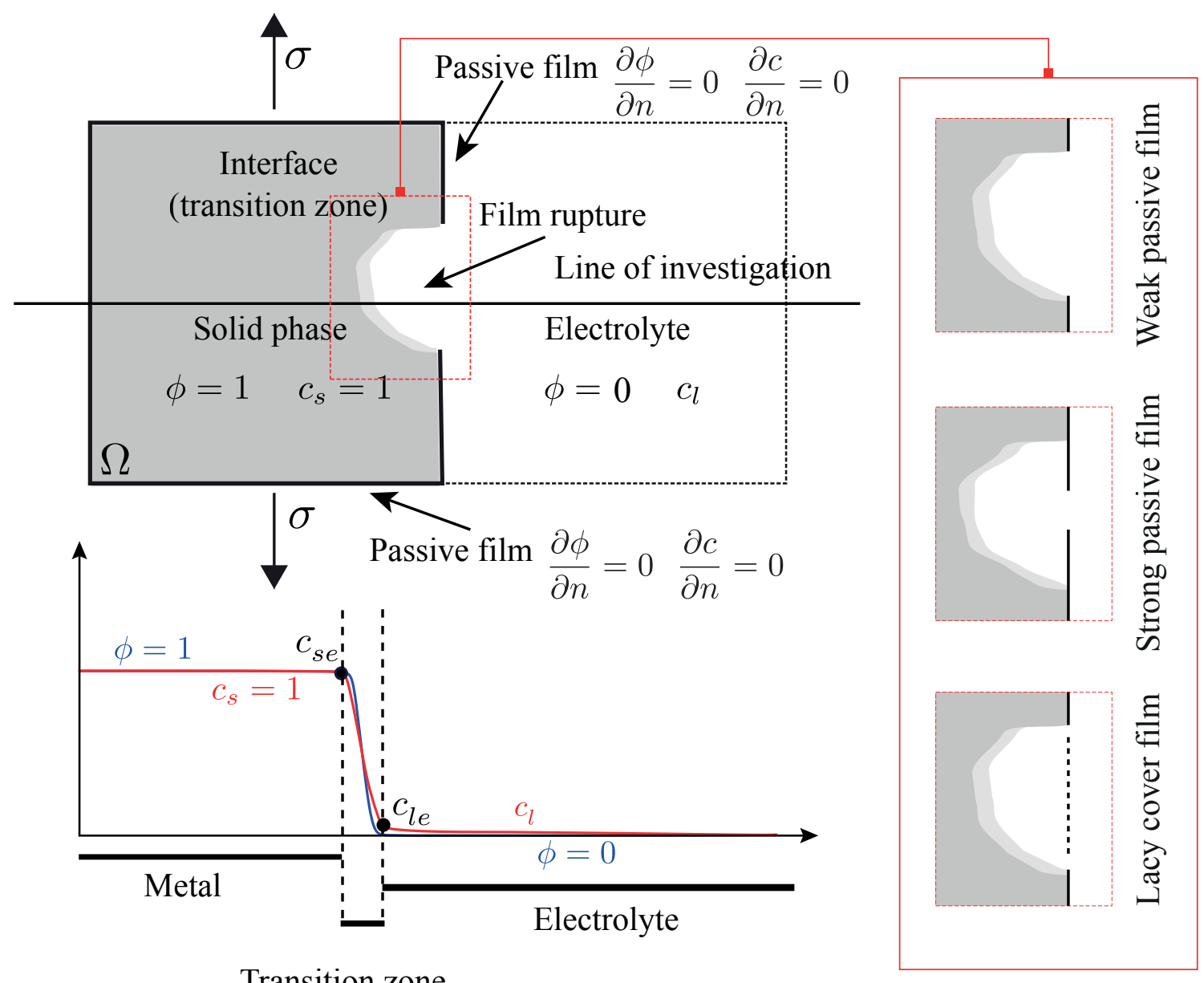

Figure 2: Schematic of a typical corrosion for different kinds of the passive film using normalized variables

\section{Phase field model}

Under the framework of variational approaches, we introduce the following energy functional for a cracked body:

$$
E(\mathbf{u}, \phi, c)=E_{u}(\mathbf{u}, \phi)+E_{c}(c, \phi)+E_{\phi}(\phi, \nabla \phi) .
$$

The term $E_{u}(\mathbf{u}, \phi)$ represents the elastic energy stored in the cracked body, $E_{c}(c, \phi)$ describes the electrochemical energy, and $E_{\phi}(\phi, \nabla \phi)$ is the energy required for material dissolution and/or crack creation. Then, the state variables are the displacement field $\mathbf{u}$, the concentration field $c$ and the phase field $\phi$.

The proposed model is based on a small strain framework incorporating three fields: displacement, concentration and phase field. Hence the energy functional contains three 
main contribution as described in Eq. 10. In this multiphysics process, there are three main interactions:

- Diffusion with mechanical and material dissolution/fracture effects

- Mechanics with diffusion and material dissolution/fracture effects

- Dissolution/fracture with diffusion and mechanical effects

However, in this work we try to capture the effect of material dissolution induced SCC by the simplest model. Hence, only the following most important factors are considered: (1) coupling between diffusion and dissolution/fracture (2) coupling between dissolution/fracture and mechanics. Note that, as the "bridging interactions" 2 , we can always capture the interactions among diffusion, mechanic and dissolution/fracture

\subsection{Elastic energy}

The elastic energy $E_{u}(\mathbf{u}, \phi)$ in the phase field framework is defined as the following:

$$
E_{u}(\mathbf{u}, \phi)=\int_{\Omega} \psi_{u}(\varepsilon(\mathbf{u}), \phi) \mathrm{d} \Omega,
$$

where $\psi_{u}(\varepsilon(\mathbf{u}), \phi)$ is the strain energy density of a damageable material. Small strain assumption is used, hence $\boldsymbol{\varepsilon}=\frac{1}{2}\left(\nabla \mathbf{u}+\nabla \mathbf{u}^{T}\right)$. To ensure that dissolution of the material is only accelerated by the tensile part of strain energy, and to prevent cracking in compression modes, we use here a unilateral contact formulation. Following the work of [32, 33, 28], the strain is decomposed into deviatoric $\varepsilon_{\text {dev }}$ and spheric $\varepsilon_{s p h}$ parts. Then, it is assumed that phase evolution (dissolution or crack propagation) is sensitive to volumetric expansion and shear.

$$
\psi_{u}(\boldsymbol{\varepsilon}(\mathbf{u}), \phi)= \begin{cases}\frac{1}{2} h(\phi)\left[\varepsilon: \mathbb{C}^{0}: \boldsymbol{\varepsilon}\right] & \text { if } \quad \operatorname{tr} \boldsymbol{\varepsilon} \geq 0 \\ \frac{1}{2}\left[\boldsymbol{\varepsilon}_{s p h}: \mathbb{C}^{0}: \boldsymbol{\varepsilon}_{s p h}\right]+\frac{1}{2} h(\phi)\left[\boldsymbol{\varepsilon}_{d e v}: \mathbb{C}^{0}: \boldsymbol{\varepsilon}_{d e v}\right] & \text { if } \operatorname{tr} \boldsymbol{\varepsilon}<0\end{cases}
$$

\footnotetext{
${ }^{2}$ An example to illustrate this property, the diffusion process will affect the dissolution/fracture and the mechanical process is directly influenced by dissolution/fracture, i.e the diffusion will affect mechanical behavior via "bridging interactions"
} 
where $\mathbb{C}^{0}$ denotes the initial elastic tensor of the material, possibly anisotropic. If $k_{0}$ denotes the corresponding bulk modulus for the undamaged material (relating the spherical part of the strain to the spherical part of the stress), hence the effective elastic tensor is:

$$
\mathbb{C}(\phi)=h(\phi) \mathbb{C}^{0}+k_{0} \mathbf{1} \otimes \mathbf{1}[1-h(\phi)] \operatorname{sign}^{-}(\operatorname{tr} \varepsilon)
$$

with the sign function $\operatorname{sign}^{-}(x)=1$ if $x<0$ and $\operatorname{sign}^{-}(x)=0$ if $x \geq 0$. The elastic density energy is now rewritten as:

$$
\psi_{u}(\varepsilon(\mathbf{u}), \phi)=\frac{1}{2}[\varepsilon: \mathbb{C}(\phi): \varepsilon]
$$

The interpolation function $h(\phi)$ can be understood as the degradation function. $h(\phi=$ $0)=0$ for fully material dissolution/damage describes the loss of material strength in this region and $h^{\prime}(\phi=0, \phi=1)=0$ ensures that the elastic energy converges to a finite value where the domain is locally intact/fully damaged.

Remark The effects of plastic strain can be easily accounted in this model by using e.g. the idea in the work [34, where the authors have proposed a new formulation of a small-strain continuum theory for brittle fracture in elasticplastic solids. It could be done by using an additive decomposition of the strain $\varepsilon$ into its elastic $\varepsilon^{e}$ and plastic $\varepsilon^{p}$ parts. In this case, contained plastic deformation can take place close to notch roots, and the new model will be a system of coupled equations governing the evolution of displacement, phase-field, concentration and accumulated plastic strain. However, for the sake of simplicity the effects of plastic deformation are not considered here. We keep it in the future work.

\subsection{Electrochemical energy}

Crack creation affected by the electrochemical process is described by the electrochemical energy $E_{c}(c, \phi)$.

$$
E_{c}(c, \phi)=\int_{\Omega} \psi_{c}(c, \phi) \mathrm{d} \Omega,
$$

where $\psi_{c}(c, \phi)$ is the electrochemical energy density. Many models have been proposed to describe this effect, e.g. the WBM (Wheeler-Boettinger-McFadden) model [35, 36], 
based on the assumption that any point within the interfacial region is assumed to be a mixture of solid and liquid both with the same composition; or under hypothesis that the transition zone is a mixture of solid and liquid with different compositions, but constant in their ratio [37]; or some others works, such as Khachaturyan's model [38] or Folch-Plapp's model [39].

In this contribution, the KKS model [11] is adopted. It postulates that the transition region is represented as a mixture of the two phases with different chemical compositions, but equal in chemical potentials:

$$
\psi_{c}(c, \phi)=h(\phi) \psi_{c s}\left(c_{s}\right)+[1-h(\phi)] \psi_{c l}\left(c_{l}\right),
$$

where $\psi_{c s}, \psi_{c l}$ are local chemical energy densities of solid metal and solution, respectively. Being evaluated in the work of [40], the expressions of $\psi_{c s}$, and $\psi_{c l}$ do not impact the evolution of coexisting phases, hence the simplest form is used as an approximation:

$$
\begin{aligned}
& \psi_{c s}\left(c_{s}\right)=A\left(c_{s}-c_{s e}\right)^{2} \\
& \psi_{c l}\left(c_{l}\right)=A\left(c_{l}-c_{l e}\right)^{2} .
\end{aligned}
$$

In these equations, $c_{s e}$ and $c_{l e}$ are the equilibrium concentrations of the coexisting phases defined in Eqs. 8,9, $A$ is the free energy density curvature which is often identified from thermodynamical databases [40, 27].

According to the KKS model [11], the condition for equilibrium at the transition zone is:

$$
\frac{\partial \psi_{c s}\left(c_{s}\right)}{\partial c_{s}}=\frac{\partial \psi_{c l}\left(c_{l}\right)}{\partial c_{l}}
$$

From (7) and (18), the expressions of concentrations in solid and solution are written as the following:

$$
\begin{aligned}
& c_{s}=c+[h(\phi)-1]\left(c_{l e}-c_{s e}\right) \\
& c_{l}=c+h(\phi)\left(c_{l e}-c_{s e}\right) .
\end{aligned}
$$

Finally, by using the Eqs. (16), (17) and (19), the electrochemical energy is properly described by two state variables, namely the phase field $\phi$ and the ions concentration $c$. 


\subsection{Surface energy}

The surface energy is the amount of energy released upon the dissolution of metal surface or the creation of new fracture surface. It is described by the following expression:

$$
E_{\phi}(\phi, \nabla \phi)=\int_{\omega} \psi_{\phi}(\phi, \nabla \phi) \mathrm{d} \Omega
$$

With $\psi_{\phi}(\phi, \nabla \phi)$ is surface energy density function, reads:

$$
\psi_{\phi}(\phi, \nabla \phi)=\omega_{\phi} g(\phi)+\alpha_{\phi}(\nabla \phi)^{2}
$$

where $g(\phi)=\phi^{2}\left(1-\phi^{2}\right)$ is the double well potential, $\omega_{\phi}$ is the height of the imposed double-well energy barrier and $\alpha_{\phi}$ is the gradient energy coefficient. In the present phase field corrosion model, $\alpha_{\phi}$ and $\omega_{\phi}$ are correlated to the interface energy $\sigma_{\phi}$ and its thickness $\ell$ of the anodic dissolution model as:

$$
\sigma_{\phi}=\frac{\sqrt{\alpha_{\phi} \omega_{\phi}}}{3 \sqrt{2}} \quad \text { and } \quad \ell=\alpha^{*} \sqrt{\frac{2 \alpha_{\phi}}{\omega_{\phi}}},
$$

where $\alpha^{*} \approx 2.94$ corresponds to the transition zone taken within the range $0.05<\phi<$ 0.95, see e.g. [41] for more details.

Note that, these two parameters ( $\omega_{\phi}$ and $\alpha_{\phi}$ ) are also directly related to the parameters of variational principle proposed to classical fracture mechanics [14, 15]:

$$
\omega_{\phi}=\frac{g_{c}}{2 \ell} \quad \alpha_{\phi}=\frac{g_{c} \ell}{2}, \text { and then } \psi_{\phi}(\phi, \nabla \phi)=g_{c}\left[\frac{g(\phi)}{2 \ell}+\frac{\ell}{2}(\nabla \phi)^{2}\right] \text {, }
$$

where $g_{c}$ is fracture energy (amount of energy dissipated upon the creation of a unit fracture surface). Using this expression of the surface energy ensures that the Griffth criterion is fulfilled. $\ell$ denotes the size of transition zone or interface between solid and liquid phases. It can be considered as a pure numerical parameter of the regularized model of brittle fracture or seen as a real material parameter for a gradient damage model. In the first case, it is recommended to take $\ell$ as small as possible to better approximate brittle fracture, with regards to the size of the mesh. In the second case, $\ell$ should be identified from experimental data. Such analysis is presently under study with experimental validations in [42, 43]. 
Remark In the present model, the surface energy implies two different physical interpretations: (SE1) energy of the material dissolution provided by electrochemical processing or mass diffusion; (SE2) energy of the fracture creation provided by mechanical loading. In the first case (SE1), the surface energy parameters are understood as the classical parameters of phase transformation model [11], including $\left(\omega_{\phi}\right.$ and $\left.\alpha_{\phi}\right)$. In the second case (SE2), the surface energy is now based on the mechanical fracture energy, defined as the product of Griffith energy $g_{c}$ with the crack surface density functional $\frac{g(\phi)}{2 \ell}+\frac{\ell}{2}(\nabla \phi)^{2}$. The definition of the surface energy allows to couple both phenomena but it could lead to some difficulties in its experimental identification. A better way is to divide into two kinds of parameter, one related to mechanical and the other one related to electrochemical processing. However, this scheme also need to define multi-phase-field (each phase-field corresponding to one process). This will change the physical meaning of damage (material dissolution/crack propagation). More investigations in future works need to be conducted on this point to make the model stronger.

\section{Variational principle}

The total energy is now rewritten as $E=\int_{\Omega} \psi \mathrm{d} \Omega$ in which:

$$
\psi=\psi_{u}+\psi_{c}+\psi_{\phi},
$$

is identified as total density energy.

\subsection{Variational principle for mechanical problem}

The displacement solution is found by solving the variational problem:

$$
\mathbf{u}(\mathbf{x})=\operatorname{Arg}\left\{\inf _{\mathbf{u} \in \mathcal{S}_{u}}\left(E(\mathbf{u}, \phi, c)-W^{e x t}\right)\right\}
$$

where $\mathcal{S}_{u}=\left\{\mathbf{u} \mid \mathbf{u}(\mathbf{x})=\overline{\mathbf{u}}\right.$ on $\left.\partial \Omega_{u}, \quad \mathbf{u} \in H^{1}(\Omega)\right\}$ and $W^{\text {ext }}=\int_{\Omega} \mathbf{f} \cdot \mathbf{u} \mathrm{d} \Omega+\int_{\partial \Omega_{F}} \overline{\mathbf{F}} \cdot \mathbf{u} \mathrm{d} \Gamma$ with $\mathbf{f}$ and $\overline{\mathbf{F}}$ body forces and prescribed traction over the boundary $\partial \Omega_{F}$. We obtain the classical weak form for $\mathbf{u}(\mathbf{x})$.

$$
\int_{\Omega} \boldsymbol{\sigma}: \boldsymbol{\varepsilon}(\delta \mathbf{u}) \mathrm{d} \Omega=\int_{\Omega} \mathbf{f} \cdot \delta \mathbf{u} \mathrm{d} \Omega+\int_{\Omega_{F}} \overline{\mathbf{F}} \cdot \delta u \mathrm{~d} \Gamma .
$$


With the above described strain energy, the Cauchy stress defined as $\boldsymbol{\sigma}=\frac{\partial \psi_{u}}{\partial \boldsymbol{\varepsilon}}$ is given by using $(12$ and 13$)$ :

$$
\boldsymbol{\sigma}=\left[h(\phi) \mathbb{C}^{0}+k_{0} \mathbf{1} \otimes \mathbf{1}[1-h(\phi)] \operatorname{sign}^{-}(\operatorname{tr} \boldsymbol{\varepsilon}(\mathbf{u}))\right]: \boldsymbol{\varepsilon}(\mathbf{u})
$$

\subsection{Variational principle for electrochemical and phase field problem}

\subsubsection{Strong form of electrochemical problem}

Cahn-Hilliard equation is used to describe the diffusion of ions from solid volume to electrolyte:

$$
\frac{\partial c}{\partial t}(\mathbf{x}, t)=\nabla \cdot M\left(\nabla \frac{\partial \psi}{\partial c}\right)
$$

where $M$ is the diffusion mobility, which can be evaluated based on an analogy between the Cahn-Hilliard equation (28) and the Fick's second law, being written as:

$$
M=\frac{D}{2 A}
$$

where $D$ is the diffusion coefficient. In this work, the diffusion coefficient $D$ is assumed to be constant for both phases (i.e $M$ is constant), so the diffusion problem to be solved to evaluate the concentration $c(\mathbf{x}, t)$ at time $t$ is:

$$
\frac{\partial c}{\partial t}(\mathbf{x}, t)=M \Delta\left(\frac{\partial \psi_{c}}{\partial c}\right)
$$

\subsubsection{Strong form of phase problem}

The phase field evolution is described by the Allen-Cahn equation:

$$
\begin{aligned}
\frac{\partial \phi}{\partial t}(\mathbf{x}, t) & =-L_{\phi}\left(\frac{\partial \psi}{\partial \phi}-\nabla \cdot \frac{\partial \psi}{\partial \nabla \phi}\right) \\
& =-L_{\phi}\left(\frac{\partial \psi_{c}}{\partial \phi}+\frac{1}{2} h^{\prime}(\phi)\left[\varepsilon: \mathbb{C}_{h}(\phi): \varepsilon\right]+\omega_{\phi} g^{\prime}(\phi)-\alpha_{\phi} \triangle \phi\right),
\end{aligned}
$$

where $\mathbb{C}_{h}(\phi)=\mathbb{C}^{0}(\mathbf{x})-k_{0}(\mathbf{x}) \mathbf{1} \otimes \mathbf{1} \operatorname{sign}^{-}(\operatorname{tr} \boldsymbol{\varepsilon}(\mathbf{x}, \tau))$ and $L_{\phi}$ is the interface kinetics parameter, that describes the transformation rate. 
In addition, to handle loading and unloading histories, we introduce the history function following the work of [16, 44]

$$
\mathcal{H}(\mathbf{x}, t)=\max _{\tau \in[0, t]}\left\{\varepsilon(\mathbf{x}, \tau):\left[\mathbb{C}^{0}(\mathbf{x})-k_{0}(\mathbf{x}) \mathbf{1} \otimes \mathbf{1} \operatorname{sign}^{-}(\operatorname{tr} \boldsymbol{\varepsilon}(\mathbf{x}, \tau))\right]: \varepsilon(\mathbf{x}, \tau)\right\}
$$

which will be instituted $\frac{1}{2}\left[\varepsilon: \mathbb{C}_{h}(\phi): \varepsilon\right]$ in 31 , yields the phase field problem to be solved to evaluate the phase field $\phi(\mathbf{x}, t)$ at time $t$ :

$$
\frac{\partial \phi}{\partial t}(\mathbf{x}, t)=-L_{\phi}\left(\frac{\partial E_{c}}{\partial \phi}+h^{\prime}(\phi) \mathcal{H}(\mathbf{x}, t)+\omega_{\phi} g^{\prime}(\phi)-\alpha_{\phi} \triangle \phi\right)
$$

\subsubsection{Weak form}

Using the variation in the concentration for Eq. (30), and the variation in the phase field for Eq. (33), the corresponding weak form is obtained:

$$
\left\{\begin{aligned}
\int_{\Omega} \frac{\partial c}{\partial t} \delta c \mathrm{~d} \Omega & +\int_{\Omega} M \nabla \frac{\partial \psi_{c}}{\partial c} \cdot \nabla \delta c \mathrm{~d} \Omega=0 \\
\int_{\Omega} \frac{\partial \phi}{\partial t} \delta \phi \mathrm{d} \Omega & +\int_{\Omega} L_{\phi}\left[\frac{\partial \psi_{c}}{\partial \phi}+h^{\prime}(\phi) \mathcal{H}(\mathbf{x}, t)+\omega_{\phi} g^{\prime}(\phi)\right] \delta \phi \mathrm{d} \Omega \\
& -\int_{\Omega} L_{\phi} \alpha_{\phi} \nabla \phi \nabla \delta \phi \mathrm{d} \Omega=0
\end{aligned}\right.
$$

\section{Numerical implementation}

Finite Element Method is used for the space discretization whereas the $\theta$-method is adopted for time integration. In order to reduce the computational cost, staggered scheme is used and two separate problems are solved: (1) electrochemical and phase field problems solved using a classical Newton-Raphson method; (2) linear equations of the displacement problem. The summary of overall algorithm is described in the following:

At time $t_{0}$ : initialize the displacement field $\mathbf{u}^{0}(\mathbf{x})$, the concentration field $c^{0}(\mathbf{x})$, the phase field $\phi^{0}(\mathbf{x})$, and the strain-history functional $\mathcal{H}^{0}=0$.

At time $t_{n+1}$ : given $\mathbf{u}^{n}(\mathbf{x}), c^{n}(\mathbf{x})$ and $\phi^{n}(\mathbf{x})$

(a) Compute the strain history functional $\mathcal{H}^{n}$, see Eq. 32

(b) Compute $c^{n+1}(\mathbf{x})$ and $\phi^{n+1}(\mathbf{x})$ by solving 34

(c) Compute $\mathbf{u}^{n+1}(\mathbf{x})$ by solving 26 . 
In the initial state, the passive film forms over metal solid, crack faces and also crack tip. SCC results from the dynamic synergy between passive film formation, crack tip strain rate, depassivation, repassivation...The slip dissolution model postulates that the rupture of passive film at the crack tip is due to the local dynamic plastic strain when the plastic strain reaches a critical value; this process allows the dissolution of metal in this region and promotes the advance of cracks. The breakdown of the passive film can be simulated by using the interface kinetics parameter $L_{\phi}$ as a function of crack tip strain rate (or in some cases creep rate) e.g. the approach proposed in the work [26]. However, the determination of strain rate is still an open question for both numerical model and experimental technique [45], which requires further efforts to make it applicable. Therefore, for sake of simplicity the breakdown of passive film is manually activated in this study and we do not consider the repassivation process.

\section{Results and discussion}

\subsection{Material parameters}

The aluminum-copper alloys are known to be prone to both Intergranular Corrosion (IGC) and Intergranular Stress Corrosion Cracking (IGSCC). IGSCC initiates from localized corrosion sites then propagation is associated to the existence of preferential anodic path along the grain boundaries, thought to be caused by either solute depleted zones or anodic precipitates [46].

Due to its X-ray absorption properties allowing easy 3D synchrotron radiation imaging and its sensitivity towards dissolution induced SCC, an aluminum alloy AA 2024-T3 was chosen for confronting experimental and numerical results. It is composed mostly of aluminum, copper and magnesium, with a typical mass density of $\rho_{\text {alloy }}=2.78 \mathrm{~g} / \mathrm{cm}^{3}$. The complete chemical composition is given in Table. 1, where the mole fraction is computed following section 2. Using Eq. 2, we obtain $\overline{\mathcal{M}}=27.747 \mathrm{~g} / \mathrm{mol}$. The metal atom concentration is then determined following Eq. 3, giving $c_{\text {solid }}^{\text {alloy }}=100.2 \mathrm{~mol} / \mathrm{l}$.

The saturated concentration is taken as in the work by [47]: $c_{s a t}=3 \mathrm{~mol} / \mathrm{l}$. Hence, the saturated concentration of solid and liquid phase are computed as in Eqs. (8), (9): $c_{s e}=1, c_{l e}=3 / 100.2$. The diffusion coefficient is assumed to be homogeneous for all 
Table 1: Chemical composition of the AA 2024-T3 alloy

\begin{tabular}{|c|c|c|c|c|}
\hline Alloy & Element & $\begin{array}{c}\text { Mass fraction, } \\
f_{m, i}[\%]\end{array}$ & $\begin{array}{c}\text { Molar mass, } \\
\mathcal{M}[\mathrm{g} / \mathrm{mol}]\end{array}$ & $\begin{array}{c}\text { Mole fraction } \\
f_{\mathcal{M}, i[}[\%]\end{array}$ \\
\hline \multirow{5}{*}{ AA 2024-T3 } & $\mathrm{Al}$ & 93.21 & 26.58 & 95.860 \\
& $\mathrm{Cu}$ & 4.36 & 63.55 & 1.904 \\
& $\mathrm{Mg}$ & 1.49 & 24.31 & 1.701 \\
& $\mathrm{Mn}$ & 0.46 & 54.94 & 0.232 \\
& $\mathrm{Fe}$ & 0.25 & 55.85 & 0.124 \\
& $\mathrm{Si}$ & 0.14 & 28.09 & 0.138 \\
& $\mathrm{Zn}$ & 0.07 & 65.38 & 0.030 \\
& $\mathrm{Ti}$ & 0.01 & 47.87 & 0.006 \\
& $\mathrm{Cr}$ & $<0.01$ & $<52.00$ & $<0.005$ \\
\hline
\end{tabular}

chemical compositions, and taken as the value for pure Al, with $D=5.41 \times 10^{-10} \mathrm{~m}^{2} / \mathrm{s}$ [47].

As mentioned in Section 3.2 , the free energy density curvature $A$ could be identified by fitting the chemical free energies obtained from Eq. 17 with ones obtained from thermodynamic databases. Following [48, 40], the chemical driving force for the phase transformation is $\Delta G=6.9 \times 10^{8} \mathrm{~J} / \mathrm{m}^{3}$. That gives $A=7.33 \times 10^{8} \mathrm{~J} / \mathrm{mol}$. (See Fig. 3).

Note that the interface energy can be either taken as the dissolution parameter related to electrochemical processing, or fracture parameter related to the mechanical loading. In this work, we chose $\sigma_{\phi}=120 \mathrm{~N} / \mathrm{m}$. Cause of lack of experimental data, the size of transition zone is here considered as a pure numerical parameter of the regularized model and taken $\ell=2.5 \mu \mathrm{m}$. The interface kinetics energy is chosen $L_{\phi}=0.15 \mathrm{l} /(\mathrm{J} . \mathrm{s})$.

For easier numerical implementation, all parameters will be normalized following the work by [40, 27]. 


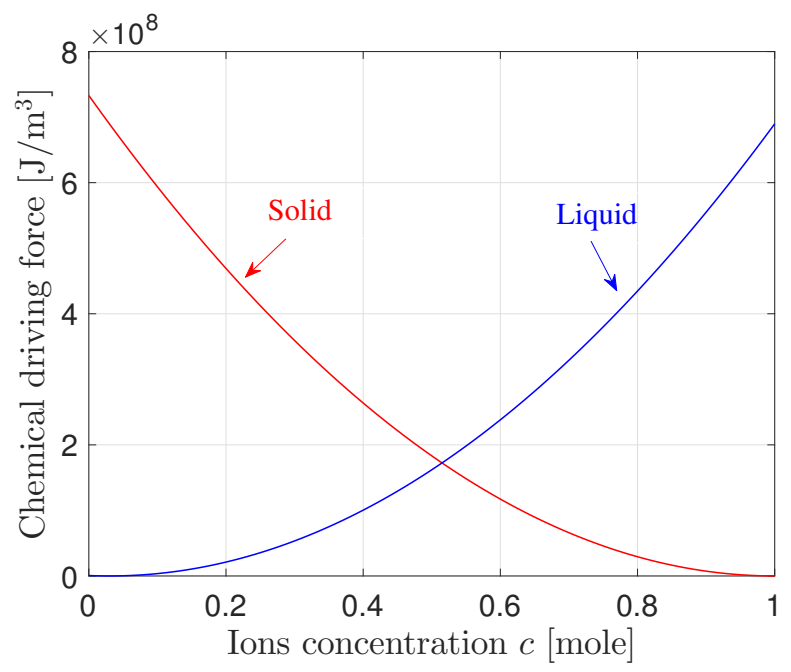

Figure 3: Variation of composition with chemical driving force fitting with thermodynamic databases.

\subsection{Case of pure local dissolution (semi-circular pitting growth)}

In the first example, the phase field model is used to simulate the localized dissolution of metal in an homogeneous structure when the passive film is broken. This mode of corrosion is described in the literature as a semi-circular pit growth. An initial semicircular shaped pit is subjected to the corrosion process. Note that only electrochemical process is considered in this first example, i.e the mechanical loading is taken null and the evolution of phase field is assumed to be isotropic for the whole simulation.

A rectangular domain with size $L \times B=200 \times 140 \mu^{2}$ is analyzed, where the film rupture region is initiated as a semi-circular area with a diameter $a=10 \mu \mathrm{m}$ on the upper edge $y=B$, as depicted in Fig. 4. In order to ensure the stability of numerical solution, the mesh size must be chosen fine enough, to have about 5-6 elements within the transition zone. Here, we take the element size $h_{e}=\ell / 10=0.5 \mu \mathrm{m}$. The material parameters are defined from the previous section 6.1.

Three kinds of passive film are investigated: (a) strong passive film, where the passive film is not dissolved together with metal; (b) the passive film is dissolved together with the metal dissolution, denoted weak passive film; (c) only half of the passive film fails and form the lacy cover. For time $t>0$, the breakdown of passive film is activated by imposing the Dirichlet boundary conditions as follows: the film rupture region is defined by $c=0$ and $\phi=0$. Neumann conditions $\frac{\partial \phi}{\partial n}=0$, and $\frac{\partial c}{\partial n}=0$ apply along the rest 


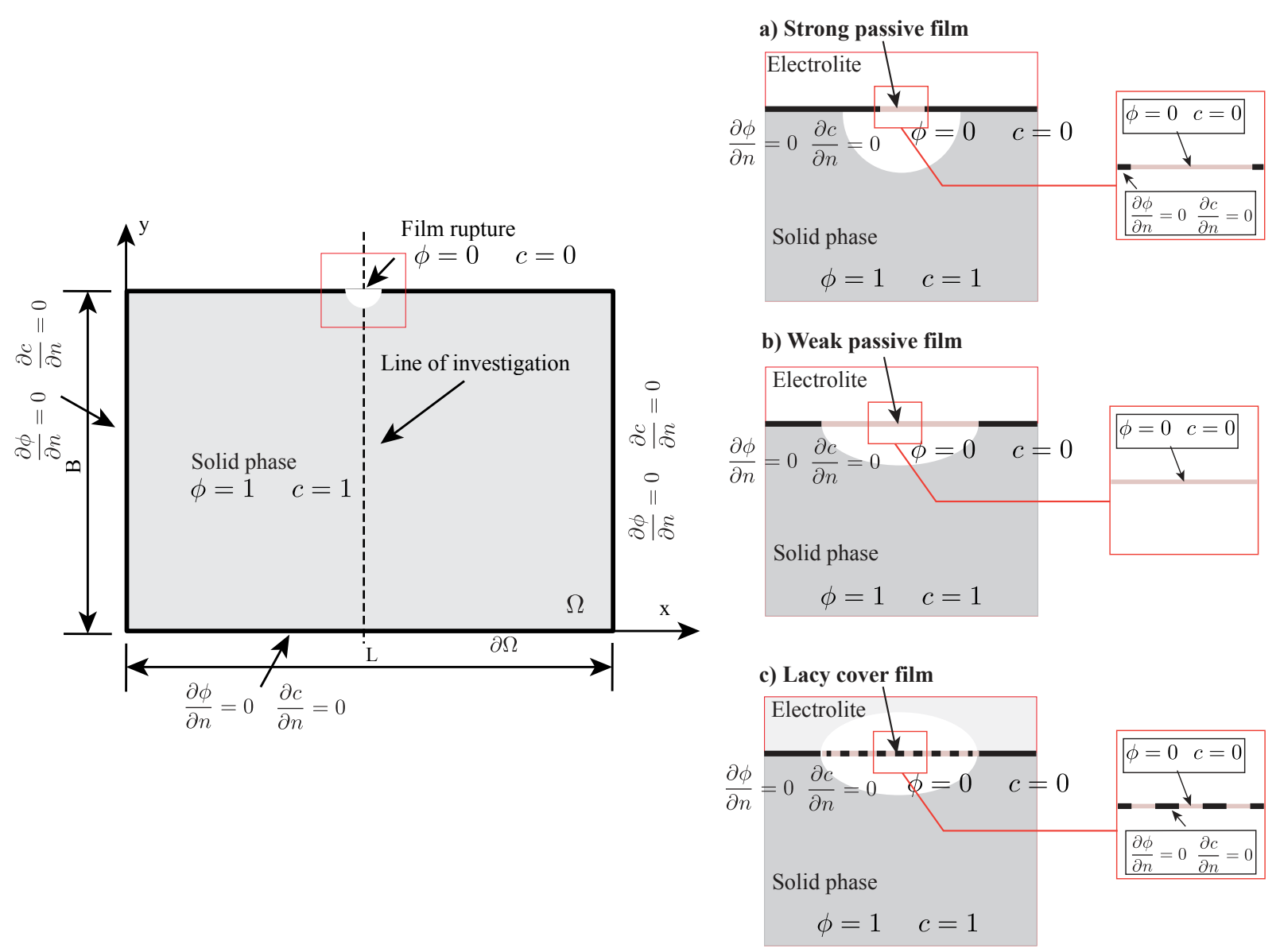

Figure 4: Geometry and boundary conditions of Benchmark test, different schemes used to model the break down of passive film is illustrated

of the domain boundary. For the strong passive film, the film rupture conditions ( $c=0$ and $\phi=0)$ are applied on a fixed region of the domain boundary. Conversely, for the weak passive condition or Lacy cover, the region where these conditions hold is updated at each time step of the analysis as illustrated in Fig. 4 (b)(c).

Figs. 5 depicts the evolution of the concentration (phase composition) and the phase field at several time steps for the first case of strong passive film. The result shows that, for the case of strong passive film, wherein it does not dissolve with metal, the pit keeps its semi-circular shape.This prediction is qualitatively in good agreement with experimental observation [49]. The concentration is recomputed in the real context (unnormalized), and then plotted along a line of investigation in (Fig. 6), The obtained results demonstrated that the ions's concentration in liquid phase is always bellow the saturation value $c_{\text {sat }}=$ 

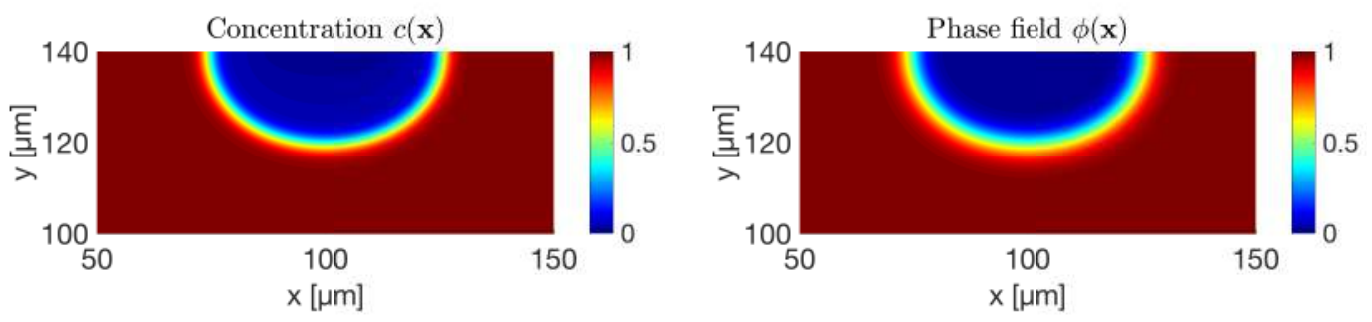

(a) $t=60 \mathrm{~min}$
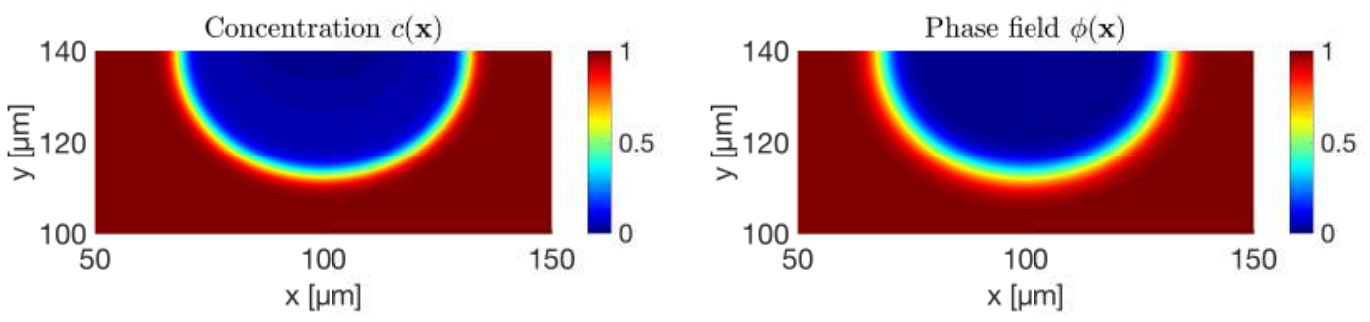

(b) $t=100 \mathrm{~min}$
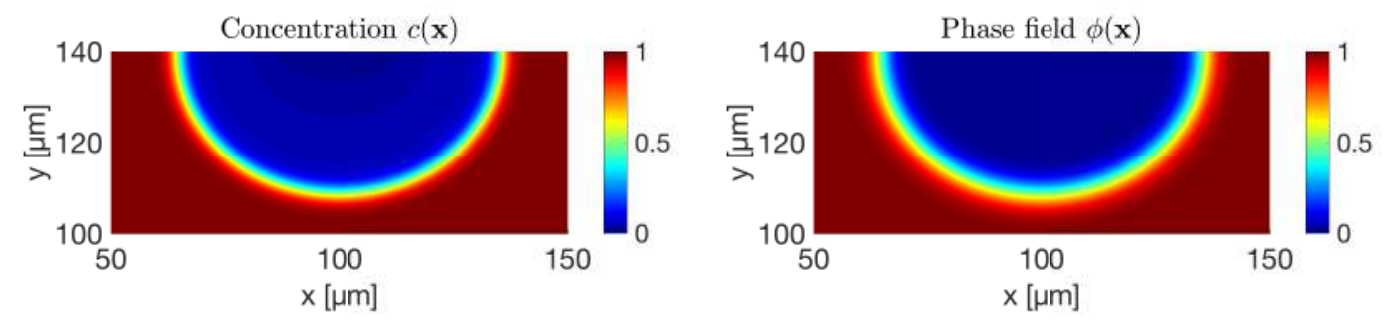

(c ) $t=140 \min$

Figure 5: Semi-circular pit growth simulation, evolution of concentration and phase field for the first case of strong passive film (size of $x, y-$ axes in $\mu \mathrm{m}$ )

$3 / 100.2$.

In the situation, where the passive is dissolved (totally for the case of weak passive film and partially for the case of Lacy cover film) along with metal, the obtained results are plotted in Fig. 7. The corrosion process is more promoted in the surface than in depth, a semi-elliptical damage form is observed. More interestingly, the Lacy cover form seems to protect the material better than weak passive film. Hence, at the same time step the case of Lacy cover passive film, the material is dissolved less than for the case of the weak passive film. These results reproduce very well th experimental observations in [49]. 


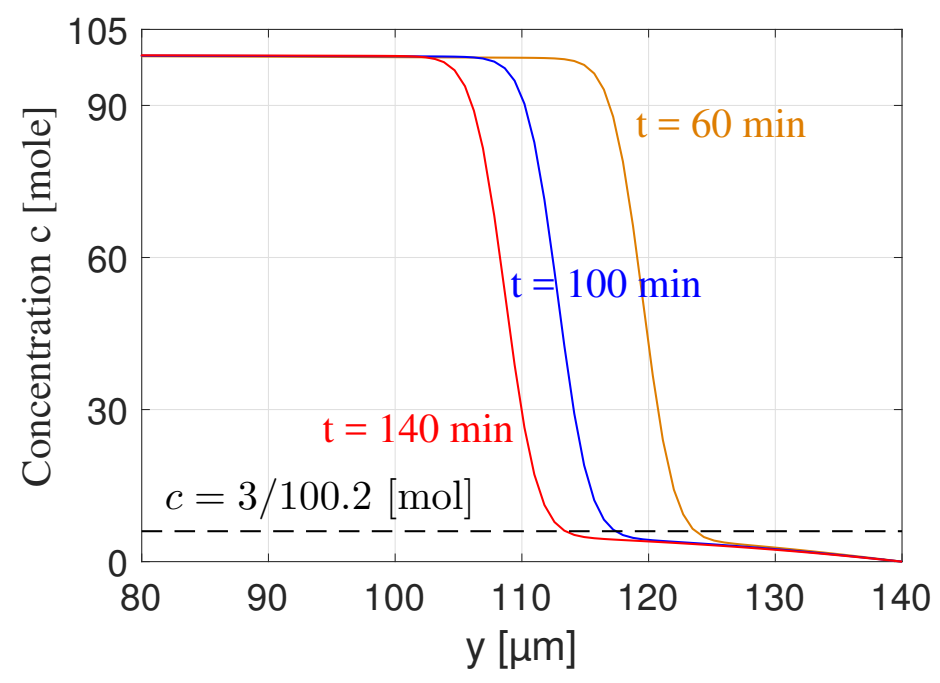

Figure 6: Evolution of real concentration (phase composition) along the line of investigation 


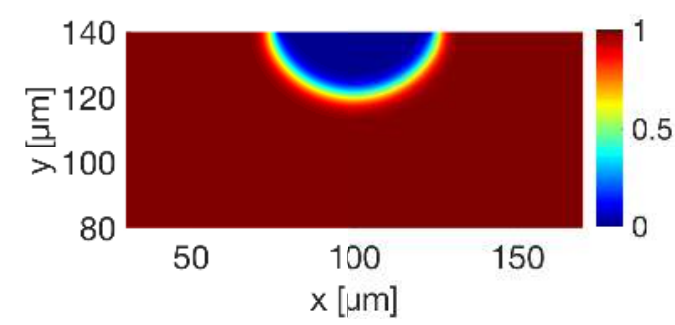

(a) weak film $t=60 \mathrm{~min}$

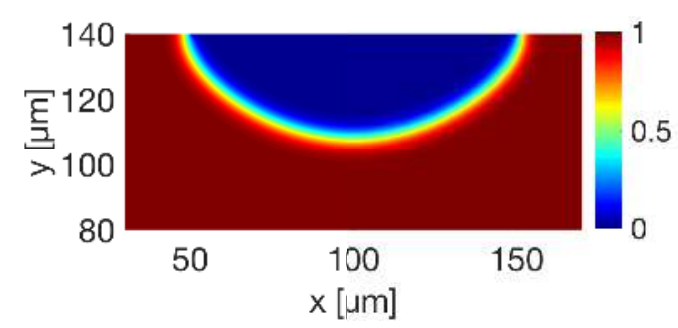

(c) weak film $t=120 \mathrm{~min}$

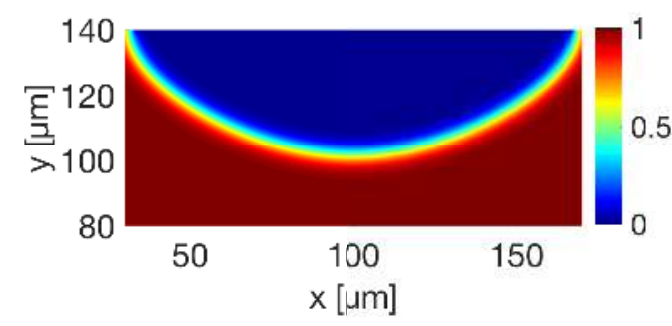

(e) weak film $t=160 \mathrm{~min}$

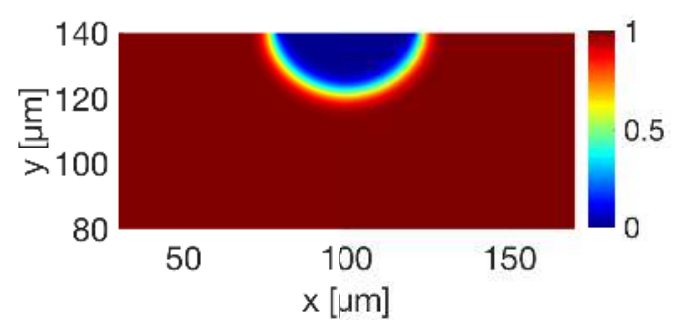

(b) Lacy film $t=60 \mathrm{~min}$

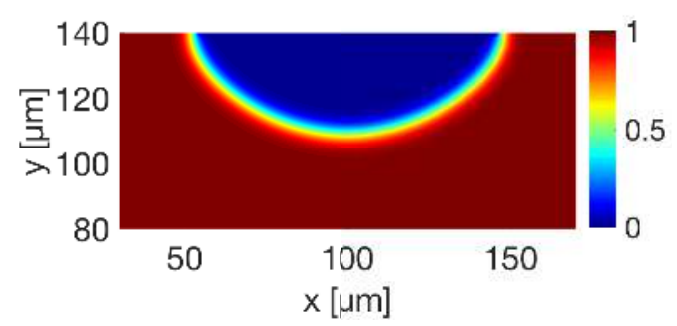

(d) Lacy film $t=120 \mathrm{~min}$

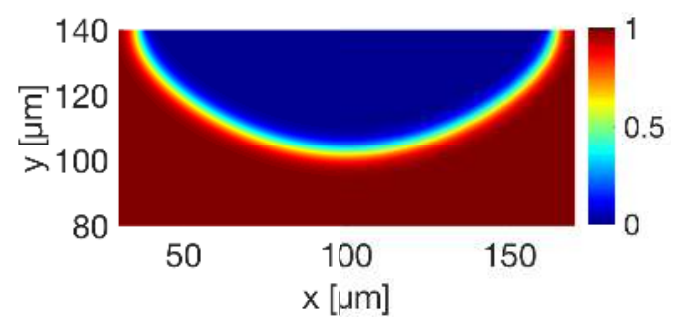

(f) Lacy film $t=160 \mathrm{~min}$

Figure 7: Corrosion morphology for the case of weak passive film (left) and Lacy cover form of passive film (right) for several time steps. The semi-elliptical damage form is observed. 


\subsection{Cases of stress corrosion cracking}

SCC tests were performed on AA 2021-T3 aluminum alloy in order to confront numerical simulation of damage growth and real propagation of stress corrosion damage, for two configurations for both numerical and experimental approaches: dissolution enhanced damage or mechanics enhanced damage.

\subsubsection{Experimental procedure and results}

The samples were machined by Electrical Discharge Machining from a $2 \mathrm{~mm}$ plate. The loading of the sample was perpendicular to the rolling direction of the plate. The sample geometry is depicted in Fig. 8.

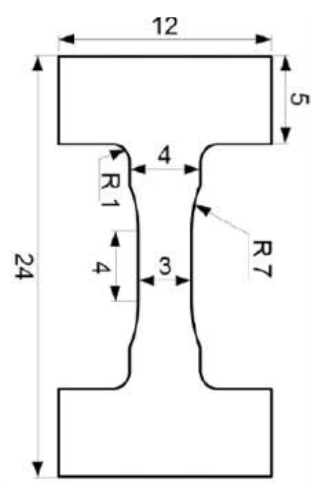

(a)

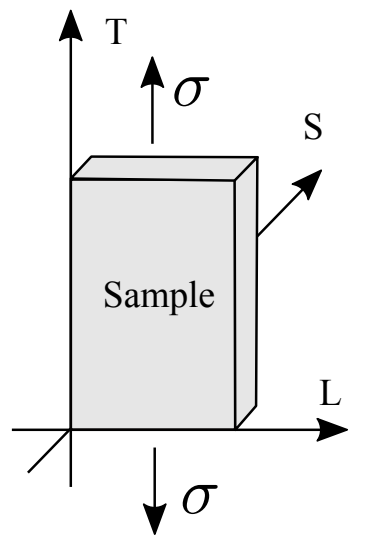

(b)

Figure 8: AA 2024 tested samples: (a) general view of sample geometry; (b) loading description

In order to obtain high resolution 3D geometrical information on cracks, in situ SCC experiments are performed on Psiche beamline at Synchrotron SOLEIL. The experimental device consists in a miniaturized tensile machine on which samples can be surrounded by a saline media ( $\mathrm{NaCl} 35 \mathrm{~g} / \mathrm{L}$ at room temperature) contained in a Perspex cylinder which also allows electrochemical measurements and control (Fig. 9).

The tensile specimen is used as the Working Electrode (WE), a saturated calomel electrode (SCE) is used as a reference and a Platinum wire as the counter electrode. Reference and counter electrodes are placed in the upper part of the cell so that they dont interact with the X-ray beam (Fig. 9). Metallic parts (grips) of the cell are protected by a varnish in order to avoid any galvanic coupling (Fig. 9). SCC initiation and propagation 


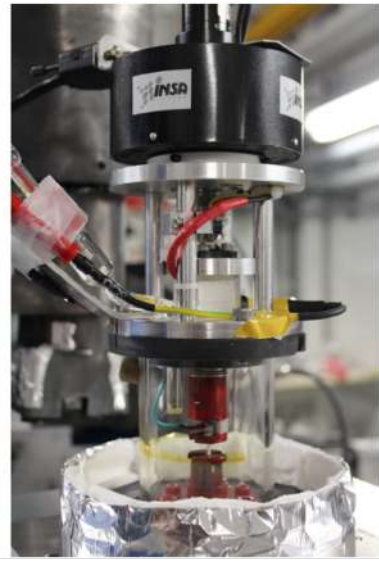

(a)

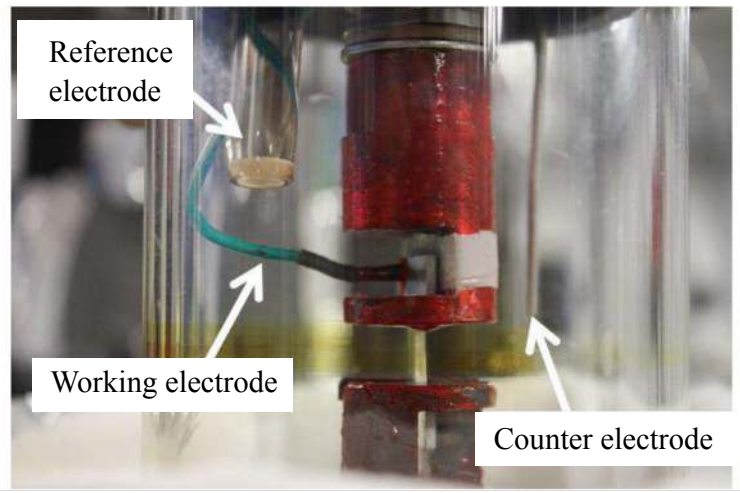

(b)

Figure 9: Experimental device: (a) general view; (b) disposition of the electrodes

are obtained by the simultaneous existence of a suitable electrochemical potential and a tensile stress.

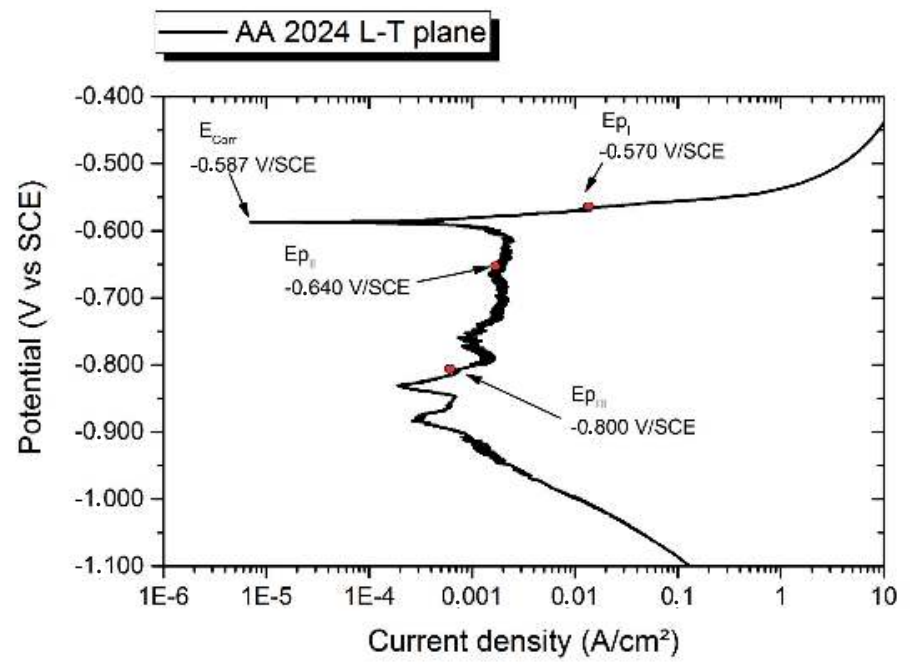

Figure 10: Polarization curve of the AA 2024 in a solution of $3.5 \%$ wt. NaCl (plane L-T)

Fig. 10 presents a polarization curve of the L-T plane of the sample in the testing solution $(3.5 \%$ wt. $\mathrm{NaCl})$. For AA 2024 alloy, SCC is associated to the existence of two pitting potentials corresponding to pitting localized at grain boundaries and within the grains respectively, the latter being higher than the former [50]. The pitting potential related to intragranular pitting can be identified as EpI in Fig. 10. On this figure, the 
identification of the pitting potential related to intergranular pitting (i.e. dissolution of the solute denuded zones along the grain boundaries [50]) is more difficult, but it can be assumed from literature results [50] that polarizing the specimen at potentials ranged between EpII and EpI will enhance intergranular pitting and SCC, whereas applying a potential equal to EpIII will prevent corrosion.

In order to enhance local dissolution, a first sample $(\mathrm{S} 1)$ is polarized at $-570 \mathrm{mV} / \mathrm{SCE}$ (EpI) from the beginning of the test. The tensile stress was applied progressively by steps of different durations from $110 \%$ to $132 \%$ of yield stress $\left(\sigma^{\text {yield }}=290 \pm 5 \mathrm{MPa}\right)$, with a strain rate of $1.25 \times 10^{-4} \mathrm{~s}^{-1}$ between each step (Fig. 11). Scans were regularly performed during deformation from $0 \%$ to $132 \%$ of yield stress. As already performed on stainless steel [51], the displacement is stopped during each scan and the potential is decreased towards less anodic domain where neither the matrix, nor the copper-depleted zone can dissolve (-800 mV/SCE (EpIII)) (Fig. 11). Images are obtained in pink beam mode $(\mathrm{E}=25 \mathrm{keV})$ on a Hamamatsu Detector $(2048 \times 2048$ pixels $)$. The scan duration is about 5 minutes (1020 images, average of 10 radiographs for each, exposure time of $25 \mathrm{~ms}$ ) with a $2.39 \mu \mathrm{m}$ voxel size. Initiations of corrosion defects are observed by tomography from the beginning of the test.

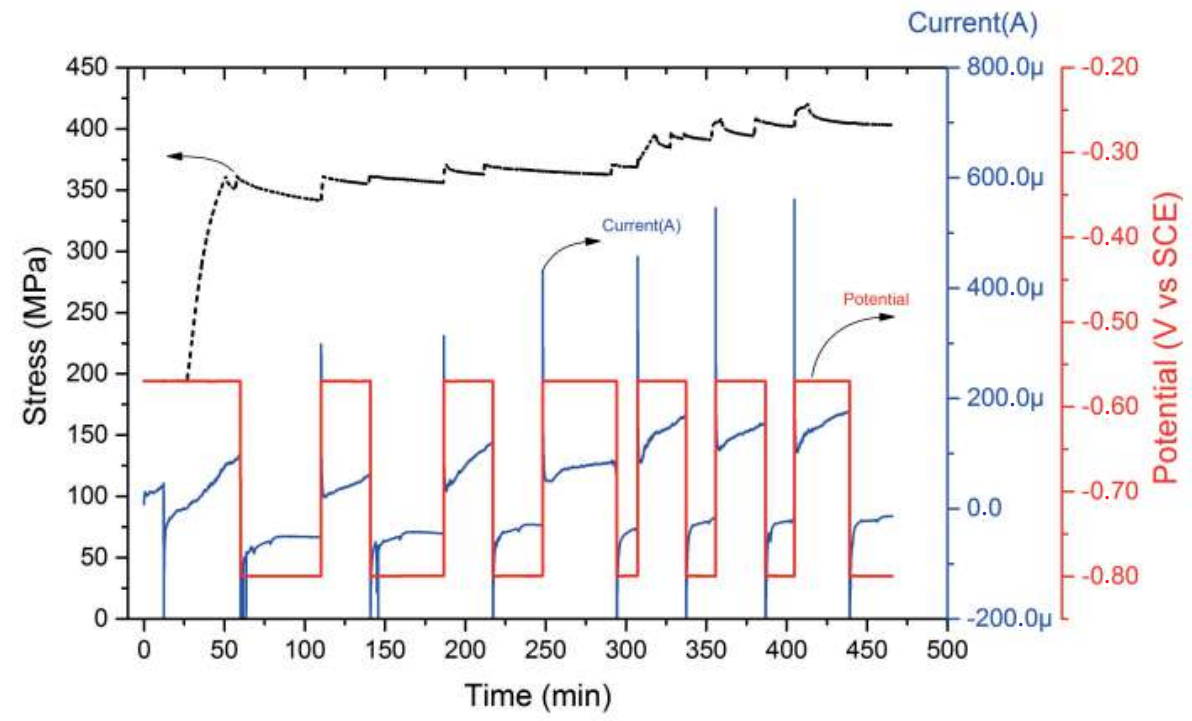

Figure 11: Loading and polarization procedures for the sample S1 (dissolution contribution enhanced) 
For the second sample, in order to promote cracking rather dissolution (pitting and/or galvanic corrosion), the potential is fixed at $-640 \mathrm{mV} / \mathrm{SCE}$ (EpII) and the stress is fixed at $125 \%$ of yield stress in the first part of the test. During this first stage, the dissolution processes should therefore be limited. No corrosion damage is actually observed. Then the potential is progressively increased up to $-570 \mathrm{mV} / \mathrm{SCE}(\mathrm{EpI})$ and the tensile stress up to $132 \%$ of yield stress (Fig. 12). In this second stage, the first cracks can be observed as soon as the final conditions of potential and stress are reached. The procedure for scanning was the same than for sample S1.

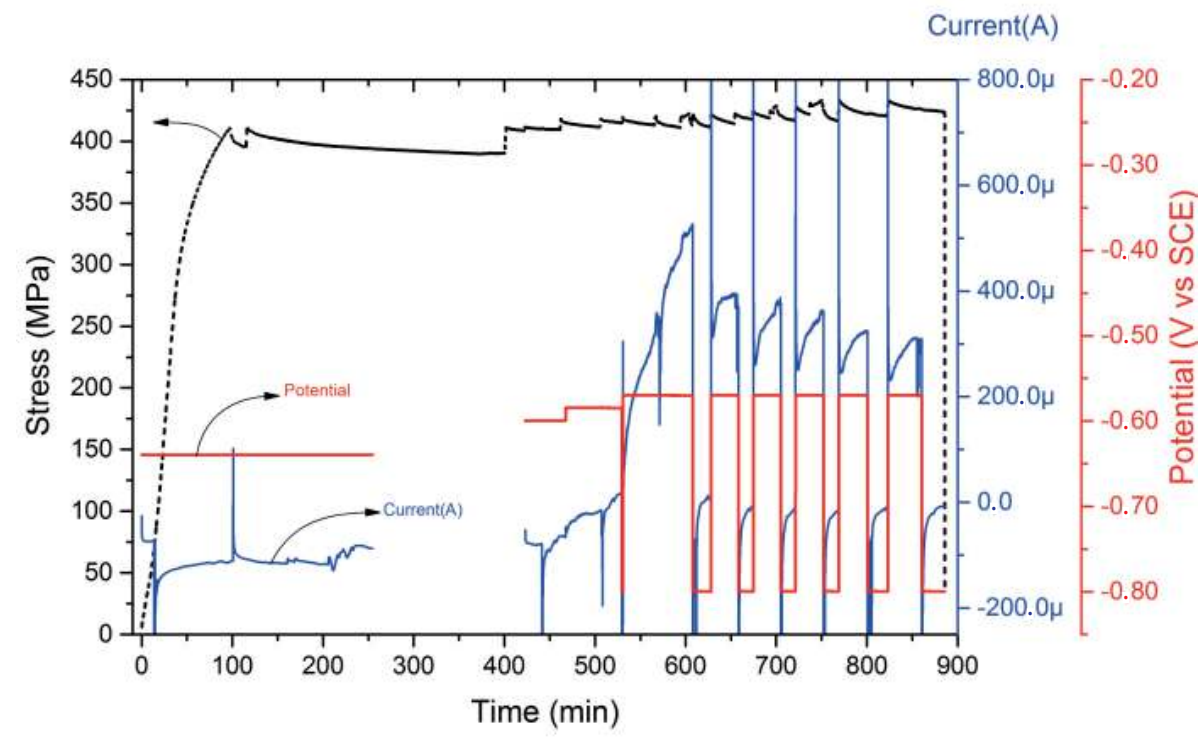

Figure 12: Loading and polarization procedures for the sample S2 (mechanical contribution enhanced)

Results are exploited at 130\% of yield stress and summarized in Fig. 13 for specimen S1, for which the dissolution is enhanced. The resulting "cracks" are shallow and branched. For the second case (specimen S2), the results are depicted in Fig. 14. It is observed that the cracks are longer and tighter than for sample S1. 

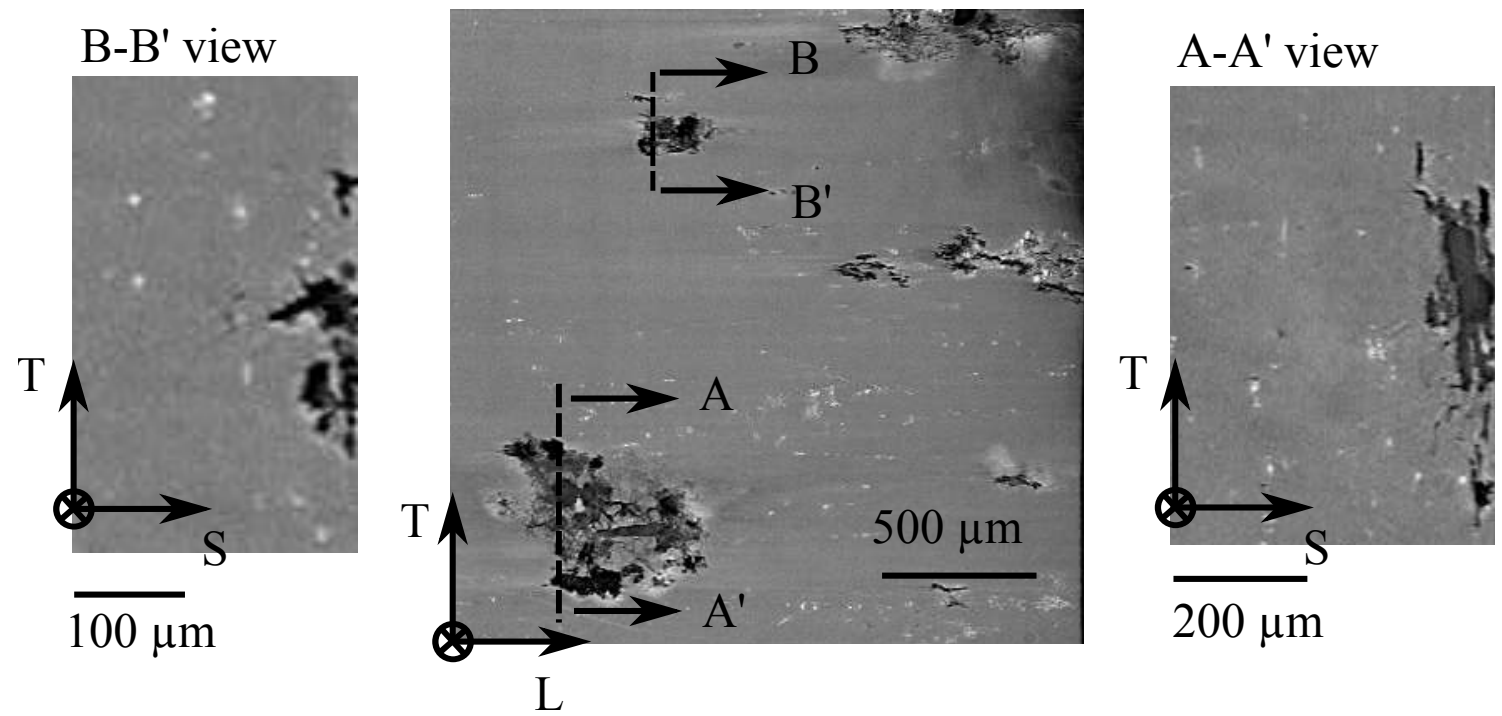

Figure 13: Morphology of the corrosion damage in the T-L and T-S directions for specimen S1 (scan performed at $130 \%$ of Yield Stress after $7 \mathrm{~h}$ of test)
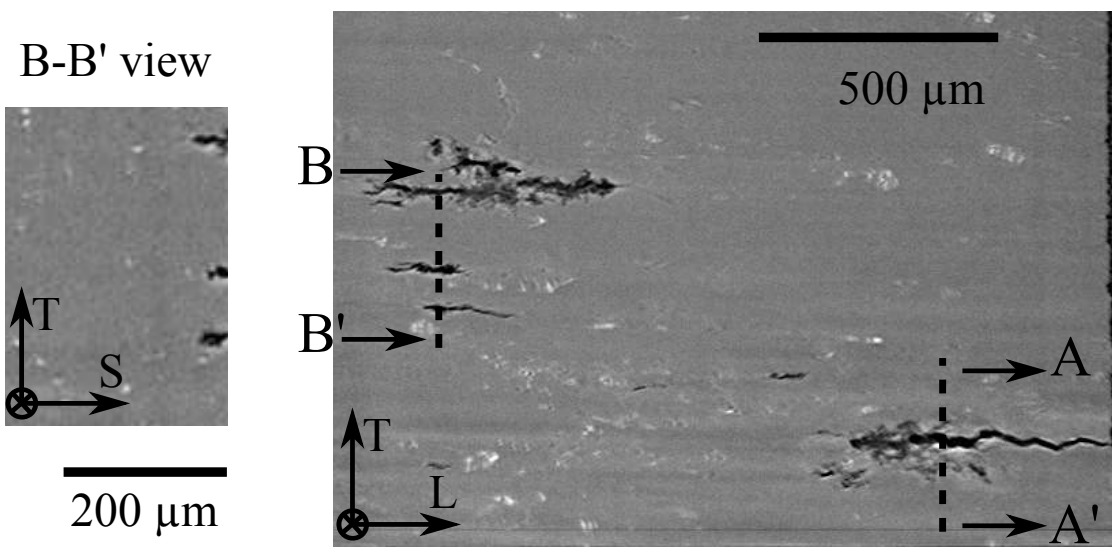

A-A' view

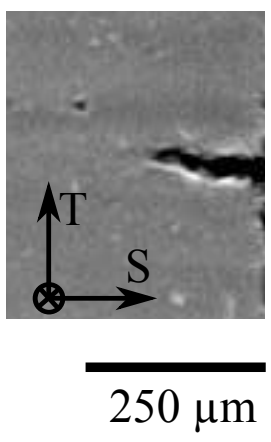

Figure 14: Morphology of the cracks in the T-L and T-S directions for specimen S2 (scan performed at $130 \%$ of Yield Stress after 10h of test) 


\subsubsection{Investigation of electrochemical and mechanical effects on SCC phenomena by using numerical approach}

In this section, the proposed model is used to simulate the relative role of electrochemical and mechanical processes on cracking. We consider a rectangular domain $L \times B=200 \times 150 \mu^{2}$ containing an initial crack, subjected to stress corrosion. Note that the crack initiation can be evidently simulated by the phase field model. However, it has been known for a long time that the fault of microstructure at smaller scale lead to stress concentration, break down the passive film and provide the crack initiation. In our case, we do not have experimental data of microstructure. Hence, the simulation of crack nucleation/initiation can not be done and the notch is introduced in the investigated structure. The details of the considered system are depicted in Fig. 15.

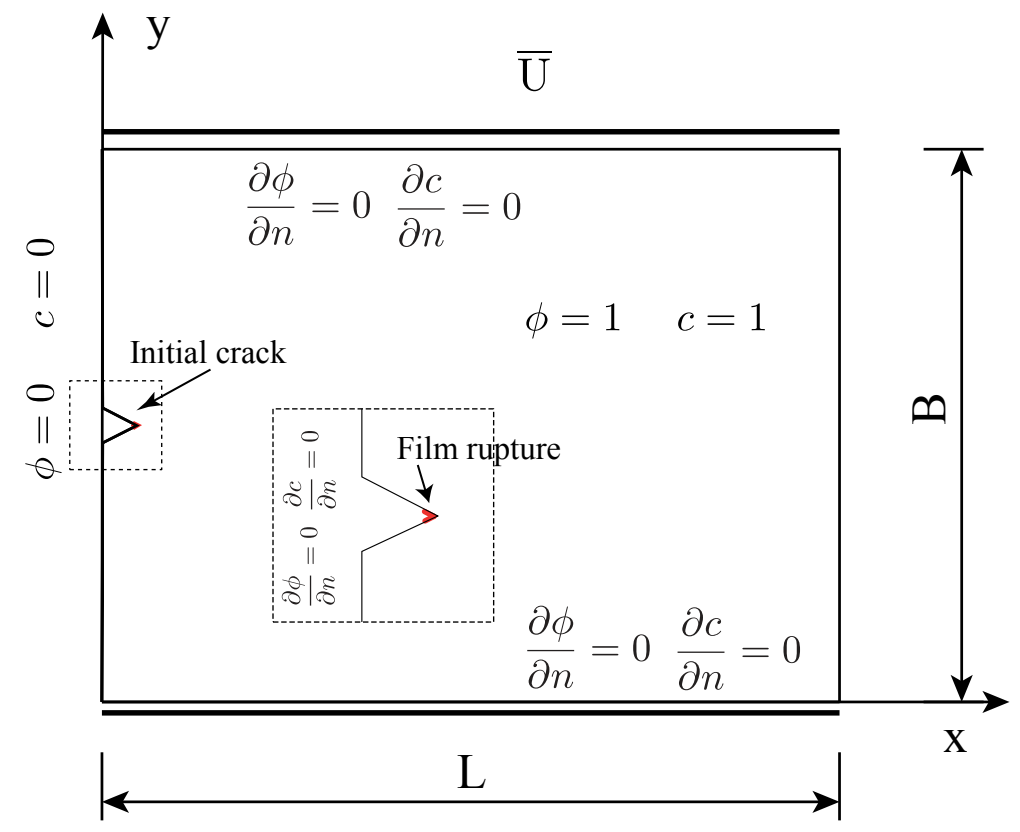

Figure 15: Geometry and boundary conditions of the investigated domain for studying related effect of electrochemistry and mechanics

The structure will be numerically assigned to both electrochemical degradation and mechanical loading according to the previous experiments. The mechanical loading (ydisplacements) is applied along the upper edge $(y=B)$. On the other hand, along the lower edge $(y=0)$, the $y$ - displacements are fixed. Along these edges, $x$ - displacements are free. The investigated structure is assumed to be covered by a strong passive film. 
The rupture of passive film allowing electrochemical processes is manually activated from the beginning at the initial crack location. Two cases are investigated:

- (C1) Electrochemical process prevails on mechanical loading (according to the sample $(\mathrm{S} 1))$

- (C2) Mechanical loading prevails on electrochemical process (according to the sample $(\mathrm{S} 2))$

In the first case $(\mathrm{C} 1)$, the structure is subjected to mechanical loading (until critical state) faster than in the second case (C2). Moreover, the SCC is deactivated in the first period of time, according to the loading condition of sample (S2). The numerical simulation is performed in 2D model, so that it shows a shorter of SCC processes time when compared to the experiment (3D in reality). The details of boundary conditions are given in Table 2 .

Table 2: Loading details of two situations: $(\mathrm{C} 1)$ and $(\mathrm{C} 2)$, where $V$ is the applied displacement rate for mechanical loading, SCC- $D$ is diffusion coefficient controlling the electrochemical process

\begin{tabular}{|c|c|c|c|c|c|c|}
\hline & \multicolumn{2}{|c|}{ Period $[0: 40 \mathrm{~min}]$} & \multicolumn{2}{c|}{ Period $[40: 60 \mathrm{~min}]$} & \multicolumn{2}{c|}{ Period $[60: 120 \mathrm{~min}]$} \\
\hline & $\mathrm{V}(\mu \mathrm{m} / \mathrm{s})$ & $\mathrm{SCC}-D\left(\mathrm{~m}^{2} / \mathrm{s}\right)$ & $\mathrm{V}(\mu \mathrm{m} / \mathrm{s})$ & $\mathrm{SCC}-D\left(\mathrm{~m}^{2} / \mathrm{s}\right)$ & $\mathrm{V}(\mu \mathrm{m} / \mathrm{s})$ & $\mathrm{SCC}-D\left(\mathrm{~m}^{2} / \mathrm{s}\right)$ \\
\hline$(\mathrm{C} 1)$ & 0.02 & $5.41 .10^{-10}$ & 0.03 & $5.41 .10^{-10}$ & 0.03 & $5.41 .10^{-10}$ \\
\hline$(\mathrm{C} 2)$ & 0.05 & $5.10^{-11}$ & 0.03 & Deactivated & 0.03 & $5.41 .10^{-10}$ \\
\hline
\end{tabular}

The material is supposed to be homogeneous, elastic and isotropic with $E=73.1$ $\mathrm{GPa}$ and $\nu=0.33$. The result of damage evolution for the first case (C1) is depicted in Fig. 16. The damage evolution in the first period is quite similar as in the example of pure local dissolution, i.e. the semi-circular shape remains. During the second period, when the loading is increased, the fracture morphology is sharper as expected for mechanically driven failure.

In the second case $(\mathrm{C} 2)$, when mechanical loading prevails on electrochemical processes, the damage evolution is depicted in Fig. 17. A classical crack shape (mechanical 


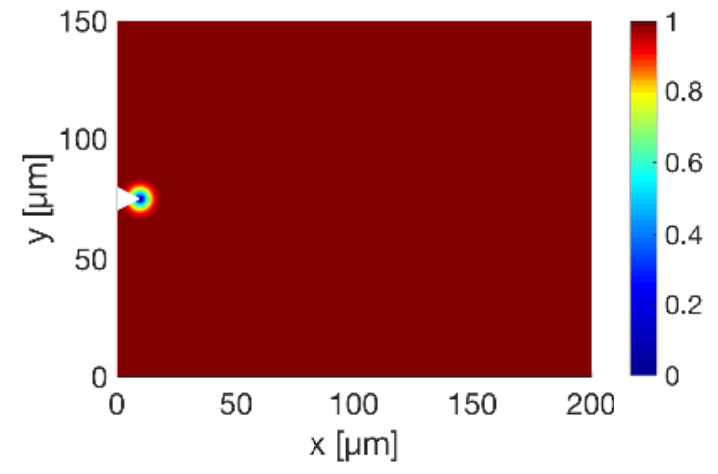

(a)

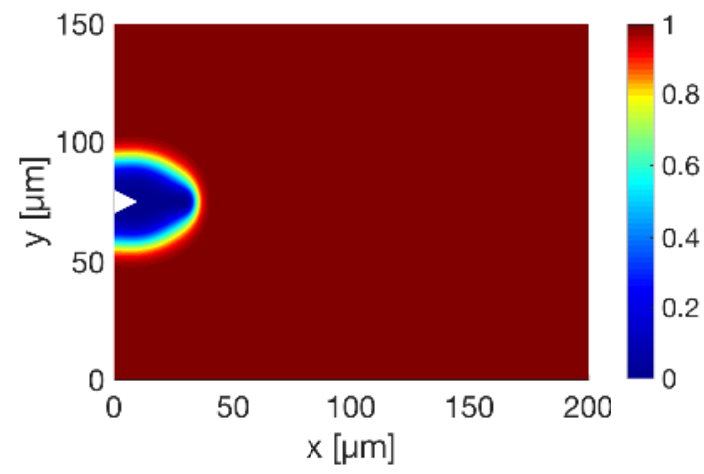

(b)

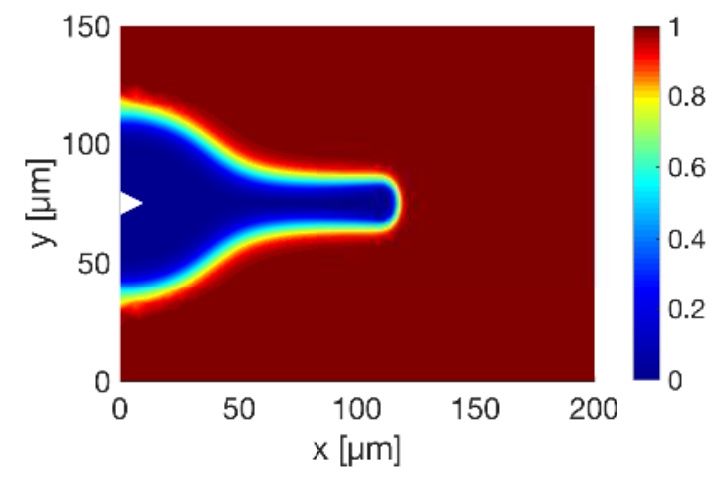

(c)

Figure 16: Case (C1) electrochemical processing prevails on mechanical loading, the damage evolution for time: $t_{a}=10 \mathrm{~min}, t_{b}=50 \mathrm{~min}$ and $t_{c}=115 \mathrm{~min}$

effect only) is obtained, but the effect of the electrochemical process is still observed. Indeed, the crack begins to propagate only from the time when the chemical process is activated and it can be noticed that the liquid domain is growing around the initial notch what is not obtained for a purely mechanical loading.

The corresponding von Mises stress is computed and plotted in Fig. 18 at a chosen loading step. In the first situation (C1), with more shallow crack, the maximal von Mises stress at crack tip is about $190 \mathrm{MPa}$. This value is much less than yield stress, i.e the damage is here mainly due to electrochemical process. For the second case $(\mathrm{C} 2)$, with shaper damage morphology, the maximal von Mises stress is observed about $320 \mathrm{MPa}$ at crack tip, which is comparable with yield stress. This result confirms the shape of the 


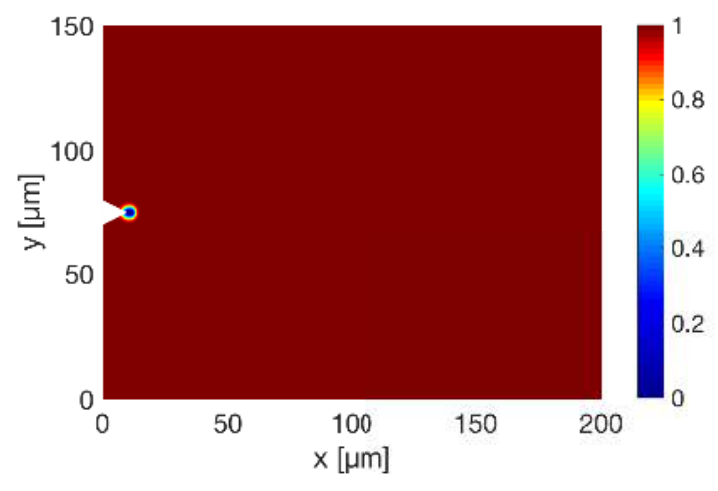

(a)

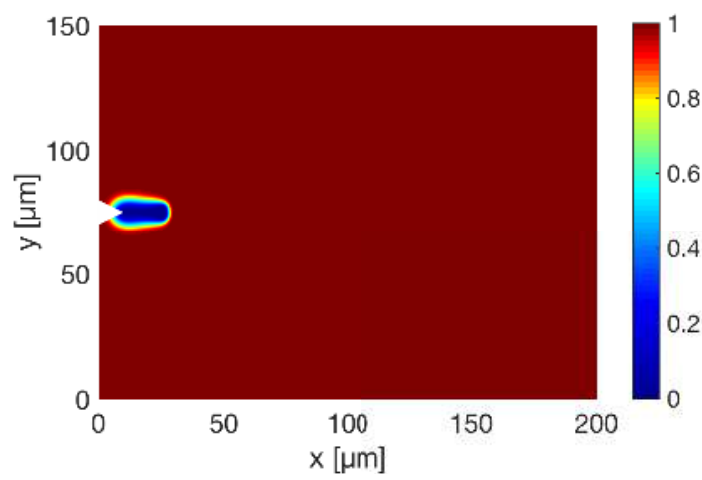

(b)

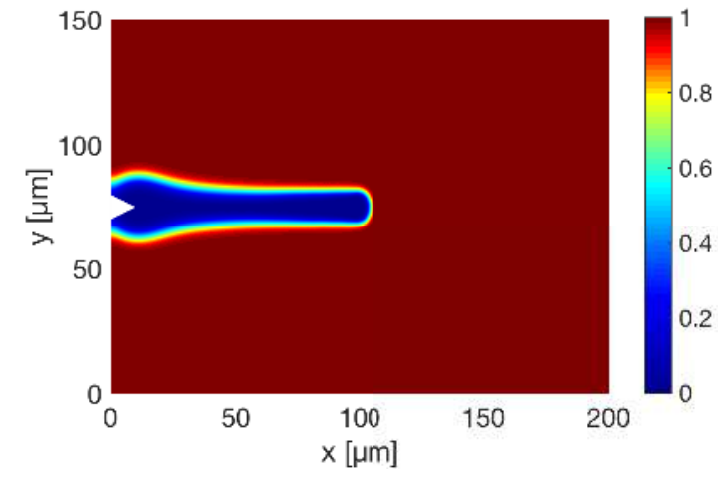

(c)

Figure 17: Case $(\mathrm{C} 2)$ mechanical loading prevails on electrochemical processing, fracture evolution for time: $t_{a}=20 \mathrm{~s} \min , t_{b}=60 \mathrm{~min}$ and $t_{c}=120 \mathrm{~min}$

damaged region (crack-likes) results from the mechanical contribution.

Figure 19 represents a detailed comparison of the damage geometry between two cases. The damage surfaces (crack edge) are extracted, showing the famous wavy-like shape as reported in the literature [52, 53]. More interestingly, when the mechanical contribution is increased, the amplitude of this wave decreases, providing a shaper damage morphology. These results are in agreement with the theoretical predictions in [52, 54]

The comparison with experimental observations in similar loading conditions is presented in Fig. 20 for the first case (C1) of the electrochemical contribution enhanced and Fig. 21 for the second situation (C2) when mechanical loading prevails on electrochemical process. Can be noted that the numerical simulations reproduce qualitatively the damage morphology obtained in the experiments for both situations. In our knowledge, that is 

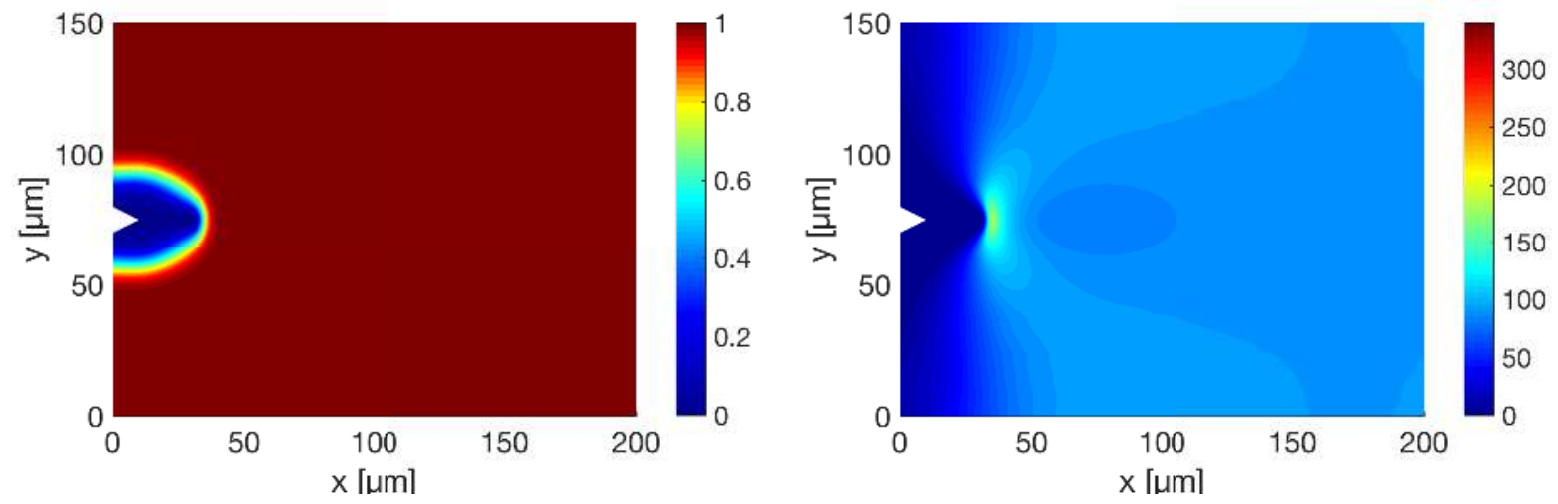

(a) damage morphology - case (C1)

(b) corresponding von Mises stress filed (C1)
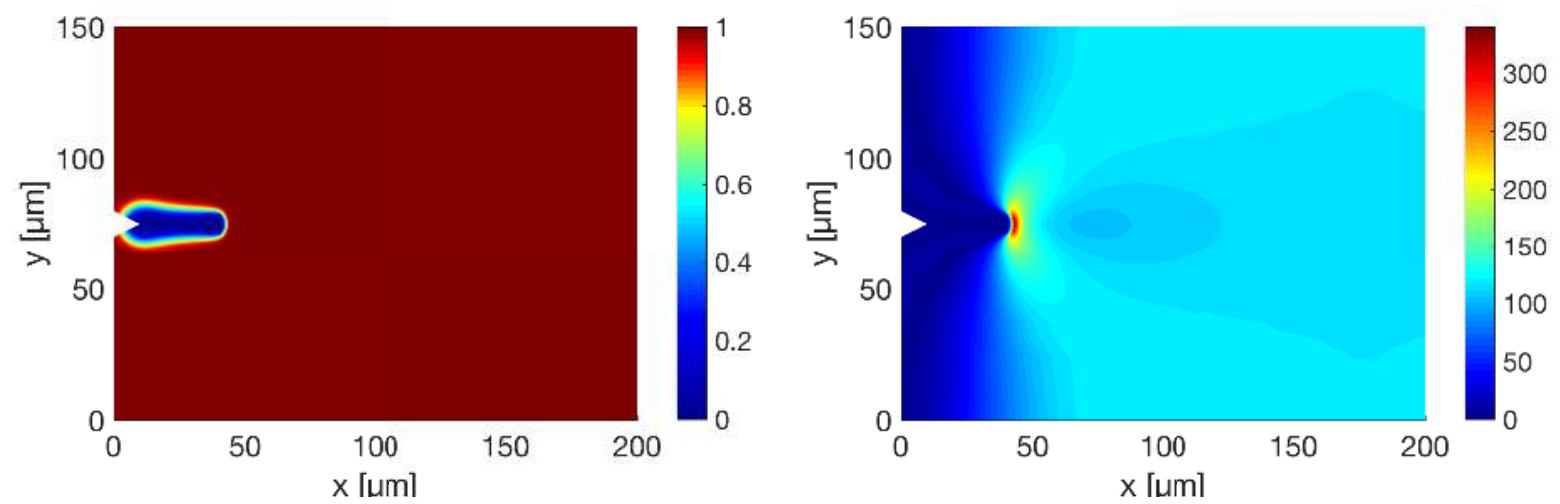

(c) damage morphology - case (C2)

(d) corresponding von Mises stress filed (C2)

Figure 18: Comparison of damage morphologies and corresponding stress states between two situations (C1) electrochemical contribution enhanced at time $t=50 \mathrm{~min}$ and $(\mathrm{C} 2)$ mechanical contribution enhanced at time $t=80 \mathrm{~min}$

done for the first time for this mode of corrosion, because neither purely electrochemical models, nor purely mechanical models can simulate the synergistic effect of stress corrosion.

The results presented in this paper are part of a wider study dedicated to simulate the coalescence of short stress corrosion cracks and involves many tests conducted on $\mathrm{Ni}$ based alloys, which were tested under yield stress. The model, based on linear elastic fracture, was developed in that context and numerical results are in very good agreement with experimental observations. Yet some limitations were encountered in the 3D characterization of cracks due to high X-Ray absorption by Ni. Some complementary tests were carried out on $\mathrm{Al}$ alloys for that reason. For these alloys, strain-hardening is necessary 


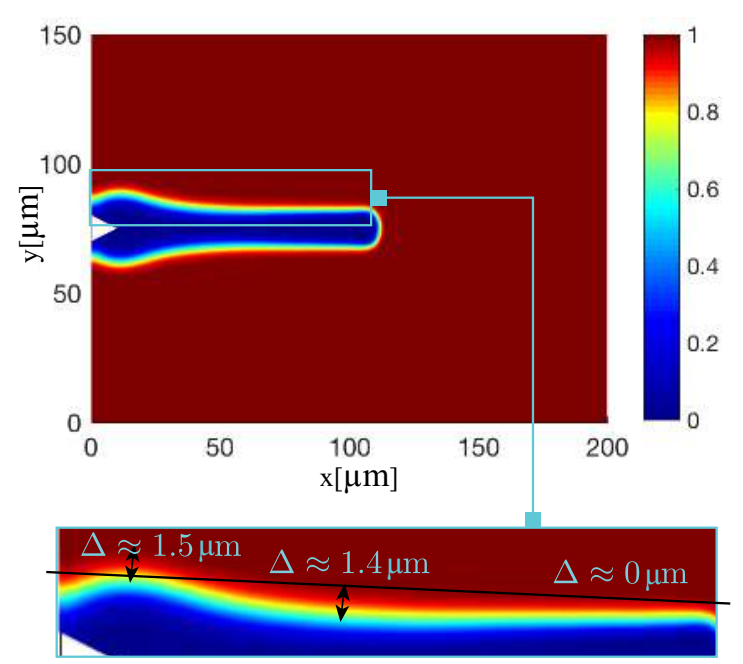

(a) damage morphology $(\mathrm{C} 2)$

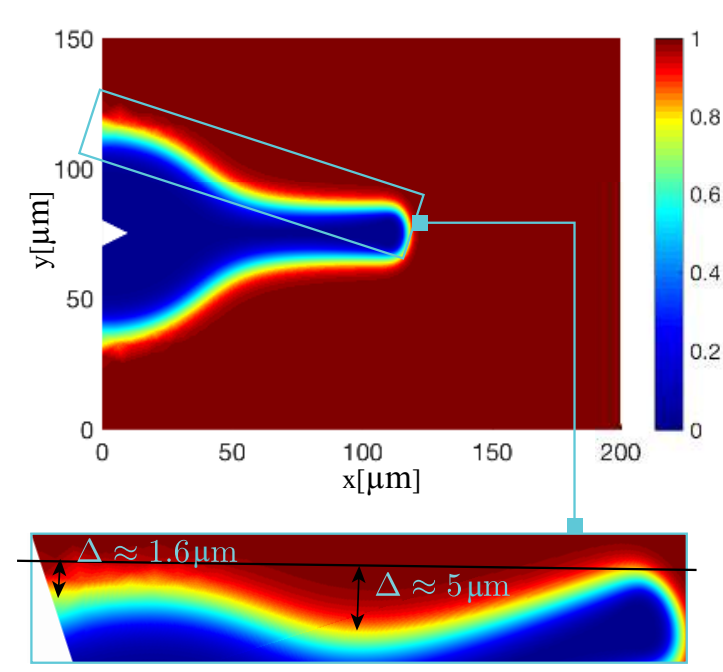

(b) damage morphology (C1)

Figure 19: Effects of mechanical and electrochemical contributions on damage phenomena. Detailed comparison of damage morphology between two situations.

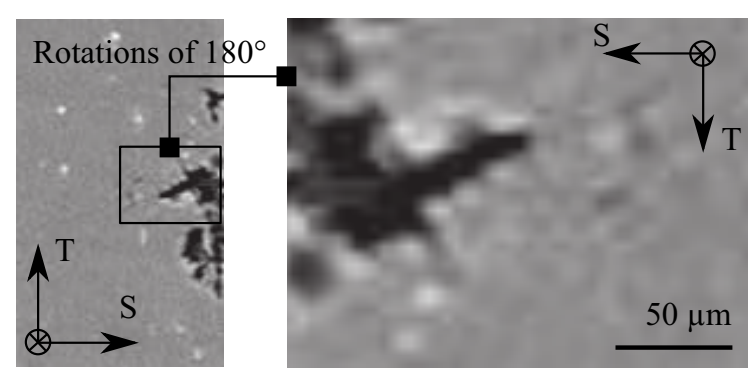

(a)

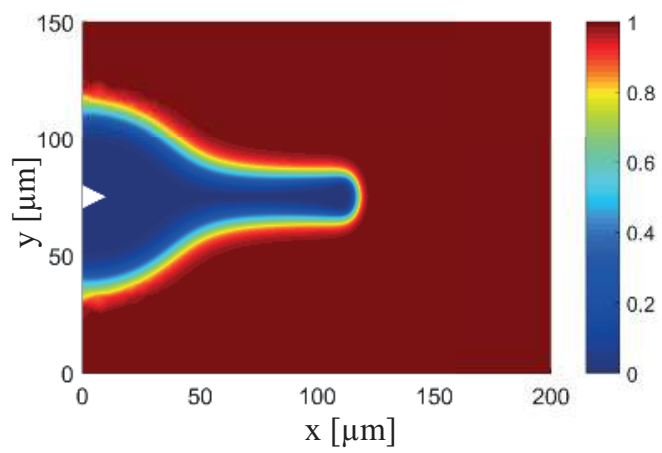

(b)

Figure 20: Case (C1) electrochemical processing prevails on mechanical loading, comparison of crack morphology of: (a) experimental observation and (b) predicted simulation

to initiate intergranular corrosion cracks in a short time (compatible with synchrotron tomography experiments). Yet, once the suitable plastic deformation is reached (132\% YS in this case), the load is maintained constant and cracks propagate with no increase of plasticity. On the other hand, the present model gives very good results for crack propagation simulation. It can therefore be concluded that if plasticity is involved in crack initiation (which is not accounted for by the model here), it is not a first order parameter 


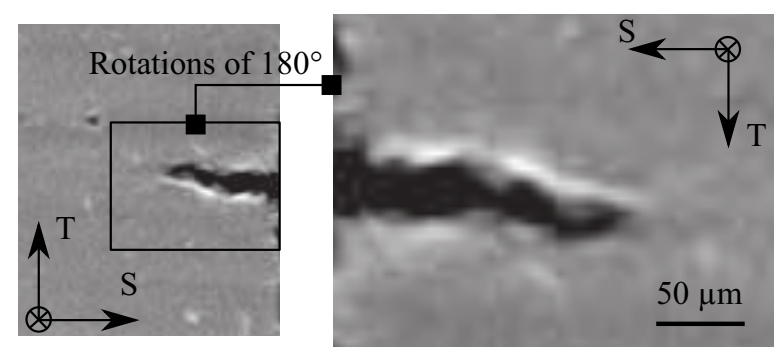

(a)

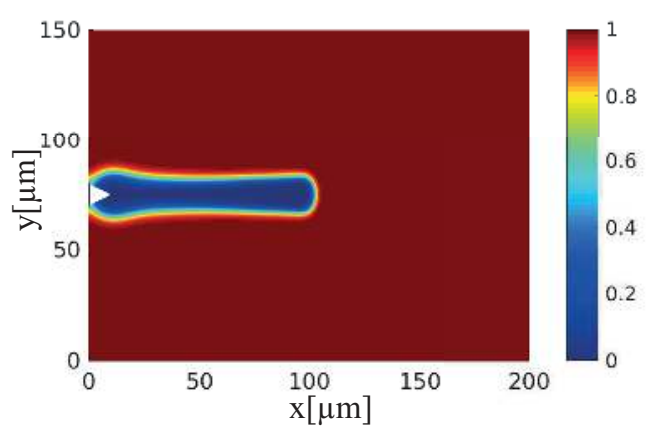

(b)

Figure 21: Case (C2) mechanical loading prevails on electrochemical processing, comparison of crack morphology of: (a) experimental observation and (b) predicted simulation

for crack propagation, which is mainly governed by dissolution.

\section{Conclusion}

A phase field framework is introduced in this work to simulate the fracture growth induced by stress corrosion. The mechanical problem is coupled with a dissolution model in a robust manner. Moreover, the impact of material anisotropy has also been incorporated leading to directional effects, possibly along several preferential directions as described for a simple situation in Appendix A. The analyses of fracture morphology provided by various SCC conditions, qualitatively agree with experimental observations. That demonstrated the performance of the proposed framework to model all aspects of SCC.

Among the prospects of this work, one can mention the further extension to the heterogeneous materials or polycrystalline systems by coupling with a cohesive model for interfaces or grain boundaries. Also, the discussion concerning the parameters of the surface energy is difficult because they could be related either to the electrochemical parameters of the dissolution model, or to the mechanical failure process. This point is still an open question and needs to be investigated for the further development of the model. Quantitative validation using experimental results is also an essential step for further developments. 


\section{Aknowledgements}

The authors would like to acknowledge the National French Research Agency (ANR) for its financial support under contract MATETPRO ANR-12-RMNP-0020 (ECCOFIC project). The authors are also grateful for the beam time awarded by synchrotron SOLEIL on the Psiche beamline (20140951 and 20150856 accepted proposals) and acknowledge warmly the technical and scientific support they benefited. The authors would like also to thank their partners Institut de la Corrosion, AREVA NP, Andra, MISTRAS Group and Pierre Combrade for their participation in the fruitful discussions during this work.

\section{Appendix A. Extending to the anisotropic behavior}

The proposed model can be easily extended to anisotropic materials. In that case, an anisotropic fracture surface density function is written by the following expression:

$$
\psi_{\phi}(\phi, \nabla \phi, \boldsymbol{\omega})=\omega_{\phi} g(\phi)+\alpha_{\phi} \boldsymbol{\omega}:(\nabla \phi \otimes \nabla \phi),
$$

where $\boldsymbol{\omega}$ is a second-order structural tensor being invariant with respect to rotations (characterizing the material anisotropy).

As considered in [33], to make the energy release rate orientation-dependent, the tensor $\boldsymbol{\omega}$ can be defined by:

$$
\boldsymbol{\omega}=\mathbf{1}+\beta(\mathbf{1}-\mathbf{N} \otimes \mathbf{N}),
$$

where $\mathbf{N}$ denotes the unit vector normal to the preferential cleavage plane (with respect to the material coordinates), and $\beta \gg 0$ is used to penalize the damage on planes not normal to $\mathbf{N}$. Hence, in the case of isotropic material $\beta=0$. Note that, for systems with many preferential directions, one phase field variable is defined for each direction. We suggest to follow the work in [28, 55] for more details.

The elastic moduli mentioned in (12) possibly depend on the material orientation. An example of the case of polycrystalline material, the rotation of the grains is taken into account to determine the elastic stiffness tensor for each grain. This can be achieved by using the reference transformation tensor and grains coordinate system. Suppose that 
the components of the elastic stiffness tensor matrix $\mathbf{C}_{g}^{0}$ (in Voigt notation) are given in grains coordinates, then the position-dependent elastic stiffness tensor with respect to the reference coordinate system is given by:

$$
\mathbf{C}^{0}=\mathbf{P}^{\mathrm{T}} \mathbf{C}_{g}^{0} \mathbf{P}
$$

where $\mathbf{P}$ is the transformation tensor in Voigt's notation.

The numerical example presented in section 6.3.2 is considered to study the influence of material orientation. The anisotropic fracture evolution described in Eqs. (A.1), A.2 is used. The penalty coefficient is $\beta=5$ and the preferential cleavage plane is oriented at $+30^{\circ}$ for $x \in[0: 75 \mu \mathrm{m}) \cup(150: 200 \mu \mathrm{m}]$ and $-30^{\circ}$ for $x \in[150: 200 \mu \mathrm{m}]$ with respect to the horizontal axis. Two sets of boundary conditions (C1) and (C2) are considered.

Figs. A.22 shows the crack propagation for both cases. In the first situation, we observe the material dissolution following the preferential plane. In the second case, the orientation dependent failure energy leads to a zigzag crack profile, which is already known in the literature, for example the work [56] on $\mathrm{MgAl}_{2} \mathrm{O}_{4}$ crystals and the work [57] on the mild, stainless steel and copper. This model is therefore promising for modelling inter and transgranular SCC at smaller scale, when the crystallographic orientation and grain boundaries have to be accounted for.

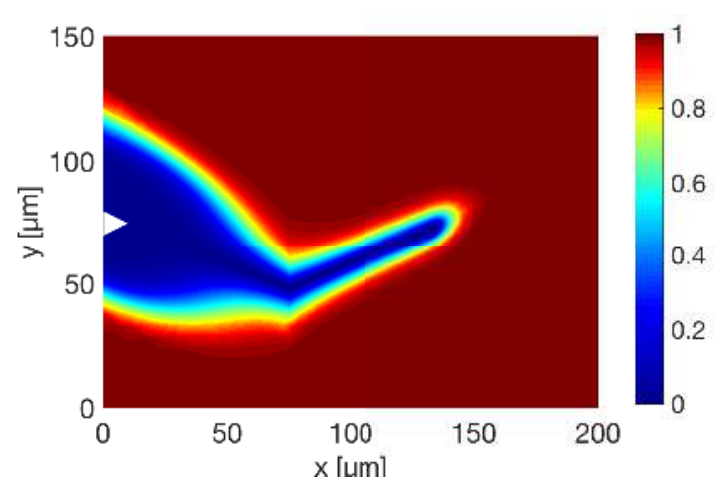

(a)

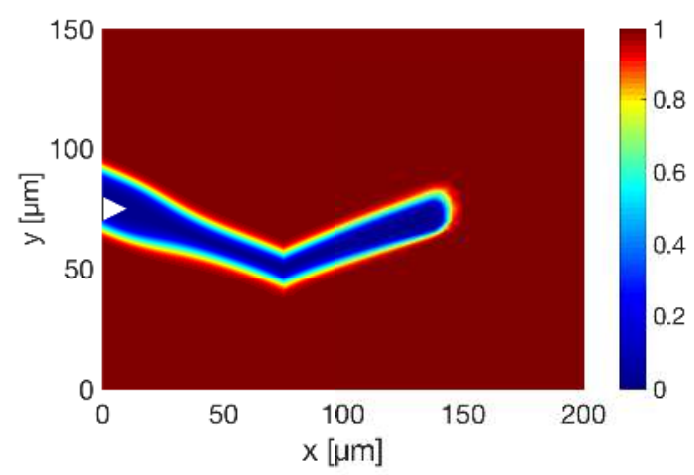

(b)

Figure A.22: Fracture morphology with anisotropic effect, the phase field is plotted corresponding to: (a) electrochemical processing prevails on mechanical loading; (b) mechanical loading prevails on electrochemical processing 


\section{References}

[1] T. Burleigh, The postulated mechanisms for stress corrosion cracking of aluminum alloys: A review of the literature 1980-1989, Corrosion 47 (2) (1991) 89-98.

[2] T. Magnin, R. Chieragatti, R. Oltra, Mechanism of brittle fracture in a ductile 316 alloy during stress corrosion, Acta Metallurgica et Materialia 38 (7) (1990) 13131319.

[3] S. Sarkar, J. Warner, W. Aquino, A numerical framework for the modeling of corrosive dissolution, Corrosion Science 65 (2012) 502-511.

[4] R. Duddu, Numerical modeling of corrosion pit propagation using the combined extended finite element and level set method, Computational Mechanics 54 (3) (2014) 613-627.

[5] N. Kota, S. Qidwai, A. Lewis, V. DeGiorgi, Microstructure-based numerical modeling of pitting corrosion in 316 stainless steel, ECS Transactions 50 (31) (2013) 155-164.

[6] W. Sun, L. Wang, T. Wu, G. Liu, An arbitrary lagrangian-eulerian model for modelling the time-dependent evolution of crevice corrosion, Corrosion Science 78 (2014) 233-243.

[7] Y. Onishi, J. Takiyasu, K. Amaya, H. Yakuwa, J. Hayabusa, Numerical method for time-dependent localized corrosion analysis with moving boundaries by combining the finite volume method and voxel method, Corrosion Science 63 (2012) 210-224.

[8] S. Gavrilov, M. Vankeerberghen, G. Nelissen, J. Deconinck, Finite element calculation of crack propagation in type 304 stainless steel in diluted sulphuric acid solutions, Corrosion science 49 (3) (2007) 980-999.

[9] C. Wojtan, M. Carlson, P. Mucha, G. Turk, Animating corrosion and erosion., in: NPH, Citeseer, 2007, pp. 15-22.

[10] R. Pidaparti, M. Palakal, L. Fang, Cellular automation approach to model aircraft corrosion pit damage growth, AIAA journal 42 (12) (2004) 2562-2569. 
[11] S. Kim, W. Kim, T. Suzuki, Phase-field model for binary alloys, Physical review e 60 (6) (1999) 7186.

[12] A. Karma, D. Kessler, H. Levine, Phase-field model of mode III dynamic fracture, Physical Review Letters 87 (4) (2001) 45501.

[13] W. J. Boettinger, J. A. Warren, C. Beckermann, A. Karma, Phase-field simulation of solidification, Annual review of materials research 32 (1) (2002) 163-194.

[14] G. Francfort, J. Marigo, Revisiting brittle fracture as an energy minimization problem, Journal of the Mechanics and Physics of Solids 46 (8) (1998) 1319-1342.

[15] B. Bourdin, G. Francfort, J. Marigo, The variational approach to fracture, Journal of Elasticity 91 (1-3) (2008) 5-148.

[16] C. Miehe, M. Hofacker, F. Welschinger, A phasefield model for rate-independent crack propagation: Robust algorithmic implementation based on operator splits, Computer Methods in Applied Mechanics and Engineering 199 (2010) 2765-2778.

[17] R. Falkenberg, Simulation of environmentally-assisted material degradation by a thermodynamically consistent phase-field model, in: Key Engineering Materials, Vol. 713, Trans Tech Publ, 2016, pp. 38-41.

[18] T. Wu, L. D. Lorenzis, A phase-field approach to fracture coupled with diffusion, Computer Methods in Applied Mechanics and Engineering 312 (2016) 196-223.

[19] Y.-H. Wen, L.-Q. Chen, J. Hawk, Phase-field modeling of corrosion kinetics under dual-oxidants, Modelling and Simulation in Materials Science and Engineering 20 (3) (2012) 035013.

[20] L. Liang, M. Stan, M. Anitescu, Phase-field modeling of diffusion-induced crack propagations in electrochemical systems, Applied Physics Letters 105 (16) (2014) 163903. 
[21] Q. Chen, N. Ma, K. Wu, Y. Wang, Quantitative phase field modeling of diffusioncontrolled precipitate growth and dissolution in Ti-Al-V, Scripta Materialia 50 (4) (2004) 471-476.

[22] I. Kovačević, B. Šarler, Solution of a phase-field model for dissolution of primary particles in binary aluminum alloys by an r-adaptive mesh-free method, Materials Science and Engineering: A 413 (2005) 423-428.

[23] Z. Xu, P. Meakin, Phase-field modeling of solute precipitation and dissolution, The Journal of chemical physics 129 (1) (2008) 014705.

[24] T.-T. Nguyen, J. Bolivar, J. Réthoré, M.-C. Baietto, M. Fregonese, A phase field method for modeling stress corrosion crack propagation in a nickel base alloy, International Journal of Solids and Structures 112 (2017) 65-82.

[25] W. Mai, S. Soghrati, R. Buchheit, A phase field model for simulating the pitting corrosion, Corrosion Science 110 (2016) 157-166.

[26] W. Mai, S. Soghrati, A phase field model for simulating the stress corrosion cracking initiated from pits, Corrosion Science 125 (2017) 87-98.

[27] A. Abubakar, S. Akhtar, A. Arif, Phase field modeling of V2O5 hot corrosion kinetics in thermal barrier coatings, Computational Materials Science 99 (2015) 105-116.

[28] T.-T. Nguyen, J. Réthoré, M.-C. Baietto, Phase field modelling of anisotropic crack propagation, European Journal of Mechanics-A/Solids 65 (2017) 279-288.

[29] S. Scheiner, C. Hellmich, Stable pitting corrosion of stainless steel as diffusioncontrolled dissolution process with a sharp moving electrode boundary, Corrosion science 49 (2) (2007) 319-346.

[30] J. Tester, H. Isaacs, Diffusional effects in simulated localized corrosion, Journal of The Electrochemical Society 122 (11) (1975) 1438-1445.

[31] Z. Chen, G. Zhang, F. Bobaru, The influence of passive film damage on pitting corrosion, Journal of The Electrochemical Society 163 (2) (2016) C19-C24. 
[32] H. Amor, J. Marigo, C. Maurini, Regularized formulation of the variational brittle fracture with unilateral contact: Numerical experiments, Journal of the Mechanics and Physics of Solids 57 (8) (2009) 1209-1229.

[33] J. Clayton, J. Knap, Phase field modeling of directional fracture in anisotropic polycrystals, Computational Materials Science 98 (2015) 158-169.

[34] F. P. Duda, A. Ciarbonetti, P. J. Sánchez, A. E. Huespe, A phase-field/gradient damage model for brittle fracture in elastic-plastic solids, International Journal of Plasticity 65 (2015) 269-296.

[35] A. Wheeler, W. Boettinger, G. McFadden, Phase-field model for isothermal phase transitions in binary alloys, Physical Review A 45 (10) (1992) 7424.

[36] A. Wheeler, W. Boettinger, G. McFadden, Phase-field model of solute trapping during solidification, Physical Review E 47 (3) (1993) 1893.

[37] J. Tiaden, B. Nestler, H.-J. Diepers, I. Steinbach, The multiphase-field model with an integrated concept for modelling solute diffusion, Physica D: Nonlinear Phenomena 115 (1) (1998) 73-86.

[38] Y. Wang, A. Khachaturyan, Shape instability during precipitate growth in coherent solids, Acta metallurgica et materialia 43 (5) (1995) 1837-1857.

[39] R. Folch, M. Plapp, Quantitative phase-field modeling of two-phase growth, Physical Review E 72 (1) (2005) 011602.

[40] S. Hu, J. Murray, H. Weiland, Z. Liu, L. Chen, Thermodynamic description and growth kinetics of stoichiometric precipitates in the phase-field approach, Calphad 31 (2) (2007) 303-312.

[41] K. Ammar, Modelling and simulation of phase transformation-mechanics coupling using a phase field method, Ph.D. thesis, École Nationale Supérieure des Mines de Paris (2010). 
[42] T.-T. Nguyen, J. Yvonnet, M. Bornert, C. Chateau, K. Sab, R. Romani, R. L. Roy, On the choice of parameters in the phase field method for simulating crack initiation with experimental validation, International Journal of Fracture 197 (2) (2016) 213226.

[43] T.-T. Nguyen, J. Yvonnet, M. Bornert, C. Chateau, Initiation and propagation of complex 3D networks of cracks in heterogeneous quasi-brittle materials: Direct comparison between in situ testing-microct experiments and phase field simulations, Journal of the Mechanics and Physics of Solids 95 (2016) 320 - 350.

[44] T.-T. Nguyen, J. Yvonnet, Q.-Z. Zhu, M. Bornert, C. Chateau, A phase field method to simulate crack nucleation and propagation in strongly heterogeneous materials from direct imaging of their microstructure, Engineering Fracture Mechanics 139 (2015) 18-39.

[45] T. Shoji, Z. Lu, H. Murakami, Formulating stress corrosion cracking growth rates by combination of crack tip mechanics and crack tip oxidation kinetics, Corrosion Science 52 (3) (2010) 769-779.

[46] D. Little, B. Connolly, J. Scully, An electrochemical framework to explain the intergranular stress corrosion behavior in two $\mathrm{Al}-\mathrm{Cu}-\mathrm{Mg}-\mathrm{Ag}$ alloys as a function of aging, Corrosion science 49 (2) (2007) 347-372.

[47] O. Guseva, J. DeRose, P. Schmutz, Modelling the early stage time dependence of localised corrosion in aluminium alloys, Electrochimica Acta 88 (2013) 821-831.

[48] T. Buhler, S. Fries, P. Spencer, H. Lukas, A thermodynamic assessment of the Al$\mathrm{Cu}-\mathrm{Mg}$ ternary system, Journal of phase equilibria 19 (4) (1998) 317-333.

[49] P. Ernst, R. Newman, Pit growth studies in stainless steel foils. i. introduction and pit growth kinetics, Corrosion Science 44 (5) (2002) 927-941.

[50] K. Urushino, K. Sugimoto, Stress-corrosion cracking of aged al-cu-mg alloys in nacl solution, Corrosion Science 19 (4) (1979) 225231-229236. 
[51] A. King, G. Johnson, D. Engelberg, W. Ludwig, J. Marrow, Observations of intergranular stress corrosion cracking in a grain-mapped polycrystal, Science 321 (5887) (2008) 382-385.

[52] R. Asaro, W. Tiller, Interface morphology development during stress corrosion cracking: Part i. via surface diffusion, Metallurgical and Materials Transactions B 3 (7) (1972) 1789-1796.

[53] P. Ståhle, E. Hansen, Phase field modelling of stress corrosion, Engineering Failure Analysis 47 (2015) 241-251.

[54] M. Grinfel'd, Instability of the equilibrium of a nonhydrostatically stressed body and a melt, Fluid Dynamics 22 (2) (1987) 169-173.

[55] T.-T. Nguyen, J. Réthoré, J. Yvonnet, M.-C. Baietto, Multi-phase-field modeling of anisotropic crack propagation for polycrystalline materials, Computational Mechanics 60 (2017) 289-314.

[56] C. Wu, K. McKinney, R. Rice, Zig-zag crack propagation in MgAl2O4 crystals, Journal of materials science letters 14 (7) (1995) 474-477.

[57] H. Kitagawa, R. Yuuki, T. Ohira, Crack-morphological aspects in fracture mechanics, Engineering Fracture Mechanics 7 (3) (1975) 515-529. 